Pacific Journal of Mathematics

SELF-ADJOINT EXTENSIONS OF SYMMETRIC SUBSPACES 


\title{
SELF-ADJOINT EXTENSIONS OF SYMMETRIC SUBSPACES
}

\author{
A. DiJksma and H. S. V. DE SNoo
}

A theory of self-adjoint extensions of closed symmetric linear manifolds beyond the original space is presented. It is based on the Cayley transform of linear manifolds. Resolvent and spectral families of such extensions are characterized. These extensions are also determined by means of analytic contractions between the "deficiency spaces" of the original symmetric linear manifold.

1. Introduction. Let $\mathfrak{F}$ be a Hilbert space over the complex numbers $C$ and denote by $\mathfrak{S}_{\mathfrak{C}}^{2}$ the Hilbert space $\mathfrak{S} \oplus \mathfrak{S}_{\mathrm{C}}$. The adjoint $T^{*}$ of a linear manifold $T$ in $\mathfrak{S}^{2}$ is a closed linear manifold defined by

$$
T^{*}=\left\{\{h, k\} \in \mathfrak{S}^{2} /(g, h)=(f, k) \text { for all }\{f, g\} \in T\right\} \text {. }
$$

A linear manifold $S$ is called symmetric if $S \subset S^{*}$ and a self-adjoint linear manifold $H$ is one for which $H^{*}=H$. Our interest will be in self-adjoint extensions of a given symmetric subspace (closed linear manifold). Such extensions were studied by Coddington in [4], who gave a complete description of all self-adjoint extensions of a symmetric linear manifold in possibly larger Hilbert spaces. In [5] Coddington applied this theory to nondensely defined ordinary differential operators and several of his results suggest that a fairly complete extension theory may be given along the lines of Naimark [7] and Straus [12].

In this paper we attempt to present such a theory thereby connecting Coddington's work to results of Phillips [10], McKelvey [6], and Schneider [11]. Fundamental is that for a self-adjoint subspace $H$ in $\mathfrak{S}^{2}$ the linear manifold $R(\lambda)=(H-\lambda)^{-1}(\lambda \in \boldsymbol{C}-\boldsymbol{R})$ is a bounded linear operator defined on all of $\mathfrak{S}$, with the properties $R(\lambda)^{*}=R(\bar{\lambda})$ and $R(\lambda)-R(\mu)=(\lambda-\mu) R(\lambda) R(\mu)$. This fact, due to Coddington [5] and also proved by Bennewitz [3], forms the basis of our paper. As was shown by McKelvey [6] and Schneider [11], these relations are sufficient to guarantee the existence of a spectral family $E(t)(t \in \boldsymbol{R})$ such that

$$
R(\lambda)=\int_{\boldsymbol{R}} \frac{1}{t-\lambda} d E(t), \lambda \in \boldsymbol{C}-\boldsymbol{R} .
$$

Thus one is led to the question what will happen when the $R(\lambda)$ 's satisfy the weaker conditions for a generalized resolvent family, 
without being injective. It turns out that they are the resolvent of an analytic family of subspaces $T(\lambda)$ in $\mathfrak{S}_{2}^{2}$ with negative imaginary part (see §3): $R(\lambda)=(T(\lambda)-\lambda)^{-1}, \lambda \in \boldsymbol{C}-\boldsymbol{R}$, while $(*)$ holds for a generalized spectral family $E(t)$. We shall present these relationships in such a way that they complete the results of McKelvey in [6].

The above results allow one to give an elementary treatment of self-adjoint subspace extensions in possibly larger Hilbert spaces of a given symmetric subspace $S$ in $\mathfrak{F}^{2}$. We introduce minimal selfadjoint extensions and show that all minimal self-adjoint extensions of $S$ with the same spectral function in $\mathfrak{S}$ are unitarily equivalent. The main theorem is that every self-adjoint extension determines a family of subspaces $T(\lambda)(\lambda \in C-R)$ in $\mathscr{S}_{2}^{2}$ such that

$$
S \subset T(\lambda) \subset S^{*} .
$$

Conversely each such family $T(\lambda)$ determines (up to unitary equivalence) a minimal self-adjoint extension of $S$. It is possible to restrict this family to a certain subspace of $\mathfrak{S}^{2}$ such that one obtains a family of operators with similar properties as the family of subspaces. Another principal result is the description of all families of $T(\lambda)$ in terms of analytic contractions between the deficiency spaces of $S$. This includes the original theorem due to Straus [12].

The Cayley transform for linear manifolds in $\mathfrak{S c}^{2}$ plays an important rôle throughout this paper. The definition of this transform given here is slightly more general than that of Arens [2]. We use the Cayley transform to analyze linear manifolds with negative imaginary part, symmetric manifolds and self-adjoint subspaces. This includes the results of Phillips [10]. Self-adjoint extensions of symmetric subspaces are also studied by means of the Cayley transform. We present some theorems which are slightly more general than the results in [4]. Coddington's description of all self-adjoint extensions in possibly larger spaces is based on the corresponding results for unitary extensions of isometric operators. Finally the Cayley transform is used in proving the subspace version of the above mentioned theorem due to Straus [12]. This is suggested by results of McKelvey in [6]. It was this last paper that formed the starting point of our work.

In $\S 2$ we give some preliminary results and a definition of the Cayley transform for linear manifolds in $\mathfrak{S c}^{2}$. Linear manifolds with negative imaginary part are analyzed in $\S 3$. Every contraction operator in $\mathfrak{F}$ is the Cayley transform of a linear manifold with negative imaginary part in $\mathfrak{S C}^{2}$, and conversely. A result due to Arens is: Every unitary operator in $\mathfrak{S}$ is the Cayley transform of a selfadjoint subspace in $\mathscr{S}^{2}$, and conversely. Our definition of maximality of a linear manifold with negative imaginary part is different from 
the one given by Phillips [10]. In terms of Cayley transforms our definition seems the more natural one. We also prove that certain (in particular self-adjoint) subspaces can be written as the orthogonal sum of a purely multi-valued part and the graph of a corresponding densely defined operator in a subspace of $\mathfrak{F}$. Section 4 is based on McKelvey's paper [6]. We study noninjective resolvents and generalized resolvents and show how they are related to certain subspaces in $\mathfrak{S E}^{2}$. We include some of McKelvey's results for completeness. Unitary extensions in a possibly larger Hilbert space of a given isometric operator are considered in $\S 5$. The description of all such extensions seems to be new. The results of the preceding sections are applied in $\S 6$, where we finally consider self-adjoint subspace extensions of a symmetric subspace.

We shall make use of results and notations as given by Coddington in [4]. We wish to thank Professor Coddington for providing us with several of his papers before publication. We understand that some years ago Professor McKelvey knew already about some of the theorems which we prove here, but did not publish them.

2. Some preliminaries. In this section we shall collect several basic observations concerning linear manifolds in $\mathfrak{S E}^{2}=\mathfrak{S E} \oplus \mathfrak{S E}$ where $\mathfrak{S E}$ is a Hilbert space. For linear manifolds $T$ and $S$ in $\mathscr{S}_{2}^{2}$ we shall use the following definitions and notations:

$$
\begin{aligned}
\mathfrak{D}(T) & =\{f \in \mathfrak{S} /\{f, g\} \in T \text { for some } g \in \mathfrak{S}\}, \\
\mathfrak{R}(T) & =\{g \in \mathfrak{S} /\{f, g\} \in T \text { for some } f \in \mathfrak{S}\}, \\
T(f) & =\{g /\{f, g\} \in T\} \text { for } f \in \mathfrak{D}(T), \\
T^{-1} & =\{\{g, f\} /\{f, g\} \in T\}, \\
\nu(T) & =\{f \in \mathfrak{S} /\{f, 0\} \in T\}, \\
T+S & =\{\{f, g\} / g=h+k \text { for some } h, k \text { such that }\{f, h\} \in T,\{f, k\} \in S\}, \\
T-\lambda & =\{\{f, g-\lambda f\} /\{f, g\} \in T\} \text { for } \lambda \in C, \\
S T & =\{\{f, k\} /\{f, g\} \in T,\{g, k\} \in S \text { for some } g \in \mathfrak{S}\}, \\
\alpha T & =\{\{f, \alpha g\} /\{f, g\} \in T\} \text { for } \alpha \in C, \\
T^{*} & =\left\{\{h, k\} \in \mathfrak{S}^{2} /(g, h)=(f, k) \text { for all }\{f, g\} \in T\right\} .
\end{aligned}
$$

In addition we shall use

$$
\begin{aligned}
T+S & =\{\{f+h, g+k\} /\{f, g\} \in T,\{h, k\} \in S\}, T \text { and } S \text { linearly } \\
\text { independent } & \\
T \oplus S & =T+S, T \text { and } S \text { orthogonal in } \mathscr{S}^{2} \\
T \ominus S & =\{\{f, g\} \in T \text { orthogonal to } S\} \\
T^{\perp} & =\mathfrak{S}^{2} \ominus T .
\end{aligned}
$$


The linear manifold $T$ is an operator if and only if $T(0)=\{0\}$. If $T$ is an operator in the traditional sense, then in this paper $T$ is frequently identified with its graph. Conversely, if $T$ is an operator in the sense of this paper then there is an operator in the traditional sense with which $T$ can be identified. The adjoint of $T$ is a closed linear manifold (subspace). Let $\bar{T}$ be the closure of $T$. Then

$$
\begin{aligned}
& T^{* *}=\bar{T}, \\
&(\alpha T)^{*}=\bar{\alpha} T^{*}, \alpha \in C, \\
&\left(T^{-1}\right)^{*}=\left(T^{*}\right)^{-1}, \\
& S \subset T \text { implies } T^{*} \subset S^{*}, \\
& \nu\left(T^{*}\right)=\Re(T)^{\perp} .
\end{aligned}
$$

For a subspace $T$ in $\mathfrak{S}^{2}$ we define $T_{\infty}$ by $T_{\infty}=\{\{0, g\} \in T\}$ and $T_{s}$ by $T_{s}=T \ominus T_{\infty}$. Then $T_{s}$ is a closed operator in $\mathfrak{S}$ with $\mathfrak{D}\left(T_{s}\right)=\mathfrak{D}(T)$.

THEOREM 2.1 (Arens). If $T$ is a subspace in $\mathfrak{S C}^{2}$, then

(i) $T_{\infty}=\{0\} \oplus T(0)$,

(ii) $T(0)=\left(\mathfrak{D}\left(T^{*}\right)\right)^{\perp}$,

(iii) $\mathfrak{D}\left(T_{s}\right)=\mathfrak{D}(T)$ is dense in $\left(T^{*}(0)\right)^{\perp}$,

(iv) $\Re\left(T_{s}\right) \subset T(0)^{\perp}$.

Proposition 2.2. If $T$ is a subspace in $\mathfrak{S}^{2}$, then $\Re(T)=\mathfrak{F}$ if and only if $\Re\left(T_{s}\right)=\mathfrak{S} \ominus T(0)$.

Lemma 2.3. Let $T$ be a subspace in $\mathfrak{S}^{2}$. Then $\Re(T)$ is closed if and only if $\Re\left(T^{*}\right)$ is closed.

Proof. Let $\Re(T)$ be closed and let $v_{n}$ be a sequence in $\Re\left(T^{*}\right)$ converging to $v \in \mathfrak{F}$ as $n \rightarrow \infty$. There exist elements $u_{n} \in \mathscr{D}\left(T^{*}\right)$ such that $\left\{u_{n}, v_{n}\right\} \in T^{*}$, hence

$$
\left(u_{n}, b\right)=\left(v_{n}, a\right)
$$

for all $\{a, b\} \in T$. This implies that $\widetilde{u}_{n}\left(\widetilde{u}_{n}\right.$ is the projection of $u_{n}$ to $\Re(T)$ ) converges weakly in the Hilbert space $\Re(T)$. Thus there exists an element $w \in \Re(T)$ such that

$$
(w, b)=(v, a)
$$

for all $\{a, b\} \in T$. Therefore $\{w, v\} \in T^{*}$ and $v \in \Re\left(T^{*}\right)$. For the proof of the converse we use the equality $T=T^{* *}$.

Lemma 2.4. Let $T$ be a linear manifold in $\mathfrak{S C}^{2}$ and $R(\lambda)=$ $(T-\lambda)^{-1}, \lambda \in C$. If $\lambda, \mu \in C$ are such that $R(\lambda)$ and $R(\mu)$ are operators defined on all of $\mathfrak{S}$, then 


$$
R(\lambda)-R(\mu)=(\lambda-\mu) R(\lambda) R(\mu),
$$

and $R(\lambda)$ and $R(\mu)$ commute.

Proof. Let $h \in \mathfrak{S}$ and $R(\mu) h=k$. Then $\{k, h\} \in T-\mu$ or $\{k, h+\mu k\} \in T$ and hence $\{k, h+(\mu-\lambda) k\} \in T-\lambda$. Thus $R(\lambda)[h+$ $(\mu-\lambda) R(\mu) h]=R(\mu) h$ for all $h \in \mathfrak{S}$.

A linear manifold $U$ in $\mathfrak{S}_{2}^{2}$ is called unitary if $U^{*}=U^{-1}$. Arens proved that a unitary subspace is an isometric operator with $\mathscr{D}(U)=$ $\Re(U)=\mathfrak{S}$.

A linear manifold $S$ is called symmetric if $S \subset S^{*}$ and a linear manifold $H$ is self-adjoint if $H=H^{*}$.

Lemma 2.5. Let $S$ be a symmetric linear manifold in $\mathfrak{S}^{2}$. If there is a number $\lambda \in C$ such that $\mathfrak{R}(S-\lambda)=\Re(S-\bar{\lambda})=\mathfrak{S}$, then $S$ is self-adjoint.

Proof. Let $\{f, g\} \in S^{*}$, then $\{f, g-\lambda f\} \in S^{*}-\lambda$. There exists an element $u \in \mathfrak{D}(S)$ such that $\{u, g-\lambda f\} \in S-\lambda$. Hence $\{f-u, 0\} \in$ $S^{*}-\lambda$. However, $\nu\left(S^{*}-\lambda\right)=(\Re(S-\bar{\lambda}))^{\perp}=\{0\}$. Thus $f=u$ or $\{f, g-\lambda f\} \in S-\lambda$ and $\{f, g\} \in S$. This shows $S^{*} \subset S$ and proves the lemma.

For a linear manifold $T$ in $\mathscr{S}_{c}^{2}$ and for $\lambda \in C$ we define the Cayley transform $C_{\lambda}$ by

$$
C_{\lambda}(T)=\{\{g-\lambda f, g-\bar{\lambda} f\} /\{f, g\} \in T\} .
$$

Then $C_{\lambda}(T)$ is a linear manifold in $\mathscr{S}_{2}^{2}$ with $\mathscr{D}\left(C_{\lambda}(T)\right)=\Re(T-\lambda)$ and $\Re\left(C_{\lambda}(T)\right)=\Re(T-\bar{\lambda})$. Note that for $\lambda \in R C_{\lambda}(T)$ reduces to the identity operator on $\Re(T-\lambda)$. The transform $F_{\lambda}$ with $\lambda \in C$ is defined by

$$
F_{\lambda}(T)=\{\{g-f, \lambda g-\bar{\lambda} f\} /\{f, g\} \in T\} .
$$

If $T$ is a linear manifold in $\mathfrak{S}^{2}$, then so is $F_{\lambda}(T)$. We have $\mathfrak{D}\left(F_{\lambda}(T)\right)=$ $\Re(T-I)$ and $\Re\left(F_{\lambda}(T)\right)=\Re(\lambda T-\bar{\lambda})$. For $\lambda \in R F_{\lambda}(T)$ reduces to multiplication by $\lambda$ on $\Re(T-I)$.

Lemma 2.6. Let $T$ and $S$ be linear manifolds in $\mathfrak{S}^{2}$ and let $\lambda \in C-$ $\boldsymbol{R}$, then ${ }^{1}$

( i ) $T=C_{\lambda}\left(F_{\lambda}(T)\right)=F_{\lambda}\left(C_{\lambda}(T)\right)$,

( ii ) $T \subset S \Leftrightarrow C_{\lambda}(T) \subset C_{\lambda}(S) \Leftrightarrow F_{\lambda}(T) \subset F_{\lambda}(S)$,

( iii ) $C_{-\lambda}(T)=C_{\lambda}(-T), F_{-\lambda}(T)=-F_{\lambda}(T)$,

(iv ) $C_{\bar{\lambda}}(T)=\left(C_{\lambda}(T)\right)^{-1}, F_{\bar{\lambda}}(T)=F_{\lambda}\left(T^{-1}\right)$,

( v ) $C_{\lambda}\left(T^{*}\right)=\left(C_{\bar{\lambda}}(T)\right)^{*}, F_{\lambda}\left(T^{*}\right)=\left(F_{\bar{\lambda}}(T)\right)^{*}$,

1 Several assertions are valid for more values of $\lambda$. 


$$
\begin{array}{ll}
\text { (vi) } C_{\lambda}(T+S)=C_{\lambda}(T)+C_{\lambda}(S), F_{\lambda}(T+S)=F_{\lambda}(T)+F_{\lambda}(S), \\
\text { (vii) } C_{\lambda}(T)(0)=\nu(T-\lambda), F_{\lambda}(T)(0)=\nu(T-I), \\
\text { (viii) } \mathfrak{D}(T)=\Re\left(C_{\lambda}(T)-I\right)=\Re\left(F_{\lambda}(T)-\lambda\right), \\
\text { (ix ) } T(0)=\nu\left(C_{\lambda}(T)-I\right)=\nu\left(F_{\lambda}(T)-\lambda\right), \\
\text { (x) } T \text { is closed } \Leftrightarrow C_{\lambda}(T) \text { is closed } \Leftrightarrow F_{\lambda}(T) \text { is closed. }
\end{array}
$$

Lemma 2.7. Let $T$ and $S$ be linear manifolds in $\mathfrak{S}^{2}$ and let $\lambda \in\{i,-i\}$, then $^{1}$
(i ) $C_{\lambda}(-T)=\left(C_{\lambda}(T)\right)^{-1}, F_{\lambda}(-T)=\left(F_{\lambda}\left(T^{-1}\right)\right)^{-1}$,
(ii) $C_{\lambda}\left(T^{-1}\right)=-\left(C_{\lambda}(T)\right)^{-1}, F_{\lambda}\left(T^{-1}\right)=-F_{\lambda}(T)$,
(iii) $T \perp S \Leftrightarrow C_{\lambda}(T) \perp C_{\lambda}(S) \Leftrightarrow F_{\lambda}(T) \perp F_{\lambda}(S)$,
(iv) $\quad C_{\lambda}(T \oplus S)=C_{\lambda}(T) \oplus C_{\lambda}(S), \quad F_{\lambda}(T \oplus S)=F_{\lambda}(T) \oplus F_{\lambda}(S)$.

3. Various linear manifolds and their Cayley transforms. A linear manifold $T$ in $\mathfrak{S C}^{2}$ is said to have the property $\operatorname{Im} T \leqq 0$ if $\operatorname{Im}(f, g) \geqq 0$ for all $\{f, g\} \in T$. The property $\operatorname{Im} T \geqq 0$ is defined analogously. Hence a linear manifold $S$ in $\mathfrak{S E}^{2}$ is symmetric if and only if $\operatorname{Im} S \leqq 0$ and $\operatorname{Im} S \geqq 0$. A linear manifold $T$ has the property Im $T \leqq 0$, if and only if for some (and hence for all) $\lambda \in C^{+2}$ and for all $\{f, g\} \in T$ the following inequality holds

$$
\operatorname{Im}(f, g-\lambda f)=(\operatorname{Im} \lambda)(f, f)+\operatorname{Im}(f, g) \geqq(\operatorname{Im} \lambda)(f, f) .
$$

A consequence of (3.1) is

$$
\|g-\lambda f\| \geqq(\operatorname{Im} \lambda)\|f\|, \lambda \in C^{+},
$$

for all $\{f, g\} \in T$.

THEOREM 3.1. Let $T$ be a linear manifold in $\mathfrak{S}^{2}$ with $\operatorname{Im} T \leqq$ 0. Then

(i) $(T-\lambda)^{-1}$ is an operator, $\lambda \in C^{+}$,

(ii) $\left\|(T-\lambda)^{-1}\right\| \leqq 1 / \operatorname{Im} \lambda, \lambda \in C^{+}$,

(iii) if $\Re(T-\lambda)$ is closed for $\lambda \in C^{+}$, then $T$ is closed.

If in addition $T$ is closed, then for all $\lambda \in \boldsymbol{C}^{+}$

(iv) $\Re(T-\lambda)$ is closed,

(v) $\Re(T-\lambda)=\left(\nu\left(T^{*}-\bar{\lambda}\right)\right)^{\perp}$,

(vi) $\Re\left(T^{*}-\bar{\lambda}\right)=\mathfrak{S}$.

Proof. The inequality (3.2) shows that

$$
(T-\lambda)^{-1}=\{\{g-\lambda f, f\} /\{f, g\} \in T\}
$$

is an operator, which satisfies (ii). Now let $\mathfrak{R}(T-\lambda)$ be closed for some $\lambda \in C^{+}$and let $\left\{u_{n}, v_{n}\right\} \in T$ converge to $\{u, v\}$ in $\mathscr{S}^{2}$ as $n \rightarrow \infty$.

1 Several assertions are valid for more values of $\lambda$.

${ }^{2}$ We shall use the notations $\boldsymbol{C}^{+}=\{\lambda \in \boldsymbol{C} / \operatorname{Im} \lambda>0\}$ and $\boldsymbol{C}^{-}=\{\lambda \in \boldsymbol{C} / \operatorname{Im} \lambda<0\}$. 
Hence $\left\{u_{n}, v_{n}-\lambda u_{n}\right\} \in T-\lambda$ converges to $\{u, v-\lambda u\} \in \mathscr{S}^{2}$ as $n \rightarrow \infty$. Thus $v-\lambda u \in \Re(T-\lambda)$ and there exists an element $w \in \mathfrak{D}(T)$ such that $\{w, v-\lambda u\} \in T-\lambda$. We apply (3.2) to the element $\left\{u_{n}-w, v_{n}-\right.$ $v-\lambda(w-u)\} \in T$ to obtain

$$
\left\|v_{n}-v\right\|+|\lambda|\left\|u-u_{n}\right\| \geqq(\operatorname{Im} \lambda)\left\|u_{n}-w\right\| .
$$

This shows $u=w \in \mathfrak{D}(T),\{u, v\} \in T$ and $T$ is closed. This proves (iii). In order to prove (iv) we let $v_{n} \in \mathfrak{R}(T-\lambda)$ converge to $v \in \mathfrak{F}$ as $n \rightarrow \infty$ for some $\lambda \in C^{+}$. Then there exist elements $u_{n} \in \mathfrak{D}(T)$ such that $\left\{u_{n}, v_{n}\right\} \in T-\lambda$. From (3.2) we obtain

$$
\left\|v_{n}-v_{m}\right\| \geqq(\operatorname{Im} \lambda)\left\|u_{n}-u_{m}\right\|,
$$

which shows that $u_{n}$ converges to some $u \in \mathfrak{F}$ as $n \rightarrow \infty$. Hence $\left\{u_{n}, v_{n}\right\}$ converges to $\{u, v\} \in T-\lambda$, since $T$ is closed. Thus $u \in \mathfrak{D}(T)$ and $v \in \Re(T-\lambda)$ and $\Re(T-\lambda)$ is closed. In general we have $(\Re(T-\lambda))^{\perp}=\nu\left(T^{*}-\bar{\lambda}\right)$, therefore the assertion (iv) implies (v). We also have $\left(\Re\left(T^{*}-\bar{\lambda}\right)\right)^{\perp}=\nu(T-\lambda)$ for $T$ is closed. The assertion (vi) follows from (i), (iv), and Lemma 2.3.

In $\S 2$ we have introduced the Cayley transform for linear manifolds in $\mathfrak{S}^{2}$. We shall now use this transform to analyse linear manifolds $T$ in $\mathscr{S}^{2}$ with $\operatorname{Im} T \leqq 0$.

THEOREM 3.2. Let $T$ be a linear manifold in $\mathfrak{S}^{2}$ with $\operatorname{Im} T \leqq 0$. If $\lambda \in C^{+}$then

(i) $C_{\lambda}(T)$ is an operator,

(ii) $\left\|C_{\lambda}(T)\right\| \leqq 1$.

Proof. Consider $C_{\lambda}(T)(0)$. If $g=\lambda f$ for $\{f, g\} \in T$ then $-(\operatorname{Im} \lambda)(f, f) \geqq 0$, which implies $f=g=0$. This proves (i). For $\{f, \mathrm{~g}\} \in T$ we have

$$
\|g-\lambda f\|^{2}=\|g\|^{2}-\lambda(f, g)-\bar{\lambda}(g, f)+|\lambda|^{2}\|f\|^{2} .
$$

Hence

$$
\|g-\bar{\lambda} f\|^{2}-\|g-\lambda f\|^{2}=-4(\operatorname{Im} \lambda) \operatorname{Im}(f, g) \leqq 0,
$$

which shows (ii).

THEOREM 3.3. Let $V$ be a linear operator in $\mathfrak{S}$ defined on $\mathfrak{D}(V)$ with $\|V\| \leqq 1$. If $\lambda \in C^{+}$then $F_{\lambda}(V)$ is a linear manifold in $\mathfrak{S}^{2}$ with $\operatorname{Im} F_{\lambda}(V) \leqq 0$.

Proof. For $h \in \mathfrak{D}(V)$ we find

$$
\operatorname{Im}((V-I) h,(\lambda V-\bar{\lambda}) h)=(\operatorname{Im} \lambda)((h, h)-(V h, V h))
$$


which proves the theorem.

The preceding theorems show that there exists a one-to-one correspondence between the linear manifolds $T$ in $\mathscr{S}^{2}$ with $\operatorname{Im} T \leqq 0$ and the linear operators $V$ in $\mathfrak{F}$ with $\|V\| \leqq 1$. We shall now consider the linear manifolds in $\mathfrak{S}_{\mathfrak{C}}^{2}$ which correspond to the linear contractions defined on all of $\mathfrak{S}$.

We shall say that the linear manifold $T$ in $\mathfrak{S}^{2}$ with $\operatorname{Im} T \leqq 0$ is maximal (denoted by $\operatorname{Im} T \leqq 0(\max )$ ) if the existence of a linear manifold $S$ in $\mathscr{S}^{2}$ with $T \subset S$ and $\operatorname{Im} S \leqq 0$ implies $S=T$. The property $\operatorname{Im} T \geqq 0(\max )$ is defined analogously.

THEOREM 3.4. Let $T$ be a linear manifold in $\mathfrak{S}^{2}$ with $\operatorname{Im} T \leqq 0$.

(i) If Im $T \leqq 0(\max )$ then $\mathfrak{R}(T-\lambda)=\mathfrak{S}$ for all $\lambda \in C^{+}$.

(ii) If $\Re(T-\lambda)=\mathfrak{S}$ for some $\lambda \in C^{+}$then $\operatorname{Im} T \leqq 0$ (max).

Proof. Let $\operatorname{Im} T \leqq 0(\max )$ and suppose $\mathfrak{D}\left(C_{\lambda}(T)\right)=\mathfrak{R}(T-\lambda)$ is not equal to $\mathfrak{S}$ for some $\lambda \in \boldsymbol{C}^{+}$. Then $C_{\lambda}(T)$ can be extended to a contraction defined on the closure of $\mathfrak{D}\left(C_{\lambda}(T)\right)$ and further extended to a contraction defined on all of $\mathfrak{S}$ in a trivial way. By Theorem 3.3 this contraction is the Cayley transform of a linear manifold $S$ in $\mathscr{S}^{2}$ with $\operatorname{Im} S \leqq 0$, such that $C_{\lambda}(T) \subset C_{\lambda}(S)$, but then by Lemma 2.6 we obtain $T \subset S, T \neq S$, which contradicts the maximality of $T$. Hence $\mathfrak{R}(T-\lambda)=\mathfrak{F}$ for all $\lambda \in C^{+}$. This proves (i). Now suppose $\mathfrak{R}(T-\lambda)=$ $\mathfrak{S}$ for some $\lambda \in C^{+}$and let $T \subset S, \operatorname{Im} S \leqq 0$. Then $C_{\lambda}(T) \subset C_{\lambda}(S)$ by Lemma 2.6 and hence $\mathfrak{D}\left(C_{\lambda}(S)\right)=\mathfrak{S}$ which shows $C_{\lambda}(T)=C_{\lambda}(S)$ but this implies $T=S$. Hence $T$ is maximal.

CoRollary 3.5. Let $T$ be a linear manifold in $\mathfrak{S}^{2}$ with $\operatorname{Im} T \leqq$ 0 (max). Then $T$ is closed.

Proof. By Theorem 3.4 we have $\mathfrak{R}(T-\lambda)=\mathfrak{H}$ for all $\lambda \in C^{+}$. The assertion of the corollary now follows from Theorem 3.1.

If $T$ is a linear manifold in $\mathfrak{S}^{2}$ it is clear that $\operatorname{Im} T \leqq 0$ if and only if $\operatorname{Im}(-T) \geqq 0$. Also $\operatorname{Im} T \leqq 0(\max )$ if and only if $\operatorname{Im}(-T) \geqq$ 0 (max).

TheOREM 3.6. Let $T$ be a subspace in $\mathfrak{S}^{2}$. Then $\operatorname{Im} T \leqq 0$ (max) if and only if $\operatorname{Im} T^{*} \geqq 0(\max )$.

Proof. Let $\operatorname{Im} T \leqq 0(\max )$. Since $\operatorname{Im} T^{*} \geqq 0(\max )$ if and only if $\operatorname{Im}\left(-T^{*}\right) \leqq 0(\max )$, it suffices to prove $\operatorname{Im}\left(-T^{*}\right) \leqq 0(\max )$. By 
Lemma 2.6 we have with $\lambda \in C^{+}$

$$
C_{\lambda}\left(-T^{*}\right)=C_{-\lambda}\left(T^{*}\right)=\left(C_{-\bar{\lambda}}(T)\right)^{*} .
$$

Since $-\bar{\lambda} \in C^{+}$it follows that $C_{-\bar{\lambda}}(T)$ is a contraction and hence so is $\left(C_{-\bar{\lambda}}(T)\right)^{*}$ so that $\operatorname{Im}\left(-T^{*}\right) \leqq 0$ by Theorem 3.3. Also we have $\Re\left(-T^{*}-\lambda\right)=\Re\left(T^{*}-(-\lambda)\right)=\mathfrak{S}$ by Theorem 3.1. The assertion follows from Theorem 3.4. Conversely $\operatorname{let} \operatorname{Im} T^{*} \geqq 0(\max )$. Since $T$ is closed $T=T^{* *}$ and we have $\operatorname{Im} T \leqq 0(\max )$ by the previous argument. This completes the proof.

THEOREM 3.7. Let $T$ be a linear manifold in $\mathfrak{S}^{2}$ and $R(\lambda)=$ $(T-\lambda)^{-1}, \lambda \in C$.

(i) If $\operatorname{Im} T \leqq 0$ (max) then for all $\lambda \in C^{+} R(\lambda)$ exists as a linear operator defined on all of $\mathfrak{K}$ and

$$
\operatorname{Im}(R(\lambda) h, h) \geqq(\operatorname{Im} \lambda)\|R(\lambda) h\|^{2}, h \in \mathfrak{S} .
$$

(ii) If for some $\lambda \in C^{+} R(\lambda)$ exists as a linear operator defined on all of $\mathfrak{S}$ and setisfies (3.3) then $\operatorname{Im} T \leqq 0$ (max).

Proof. Since $\operatorname{Im} T \leqq 0(\max )$ we have $\mathscr{D}(R(\lambda))=\Re(T-\lambda)=\mathfrak{K}$ for all $\lambda \in \mathbb{C}^{+}$by Theorem 3.4. Let $h \in \mathfrak{S}$ and $k=R(\lambda) h$ then $\{k, h+\lambda k\} \in T$. Hence

$$
0 \leqq \operatorname{Im}(h, h+\lambda k)=\operatorname{Im}(R(\lambda) h, h+\lambda R(\lambda) h)
$$

which implies (3.3). This proves (i). In order to prove (ii) we note that for the given $\lambda \in \mathbb{C}^{+} \Re(T-\lambda)=\mathfrak{D}(R(\lambda))=\mathfrak{S}$. If $\{h, l\} \in T$, then $R(\lambda)(\hbar-\lambda h)=h$. Applying (3.3) we find

$$
\operatorname{Im}(h, l \sigma-\lambda h) \geqq(\operatorname{Im} \lambda)\|h\|^{2}
$$

and this gives $\operatorname{Im}(h, h) \geqq 0$. The assertion (ii) follows from Theorem 3.4 .

THEOREM 3.8. Let $V$ be a linear operator in $\mathfrak{S}$ defined on $\mathfrak{D}(V)$ with $\|V\| \leqq 1$.

(i) If $\Re(V-I)$ is dense in $\mathscr{S}$, then $V-I$ is injective. In addition we let $\mathfrak{D}(V)=\mathfrak{S}$.

(ii) If $V-I$ is injective, then $\Re(V-I)$ is dense in $\mathfrak{S}$.

(iii) If $V_{1}$ is the restriction of $V$ to $\mathfrak{S} \ominus \nu(V-I)$, then $V_{1}$ is an operator in $\mathfrak{S} \ominus \nu(V-I)$ and $\Re\left(V_{1}-I\right)$ is dense in $\mathfrak{S} \ominus \nu(V-I)$.

Proof. For the proof of (i) we refer to Phillips [10, p. 200]. In order to prove (ii) we use a simple result which can be found in [13, p. 8]: $\nu(V-I)=\nu\left(V^{*}-I\right)$ if $V$ is a contraction. Hence 
$(\Re(V-I))^{\perp}=\nu\left(V^{*}-I\right)=\nu(V-I)=\{0\}$. This shows that $\Re(V-I)$ is dense in $\mathfrak{S}$, which is (ii). Finally we note that for all $y \in \mathfrak{F}$ and $z \in \nu(V-I)$ we have

$$
(V y, z)=(y, z) \text {. }
$$

This implies $V y \perp \nu(V-I)$ for all $y \in \mathfrak{S} \ominus \nu(V-I)$.

COROLLARY 3.9. Let $T$ be a linear manifold in $\mathfrak{S}^{2}$ with $\operatorname{Im} T \leqq$ 0. If $\mathfrak{D}(T)$ is dense in $\mathfrak{B}$, then $T$ is an operator.

Proof. It follows from Lemma 2.6 that $\mathfrak{R}\left(C_{\lambda}(T)-I\right)=\mathfrak{D}(T)$ is dense in $\mathfrak{S}_{\text {. F F }} \lambda \in C^{+}$Theorem 3.8 implies that $C_{\lambda}(T)-I$ is injective. However, by Lemma 2.6 we have $\nu\left(C_{\lambda}(T)-I\right)=T(0)$, hence we find $T(0)=\{0\}$.

Let $T$ be an operator in $\mathfrak{S}$ with $\operatorname{Im} T \leqq 0$. We shall say that $T$ is operator maximal if the existence of an operator $S$ in $\mathfrak{S}$ with $T \subset S, \operatorname{Im} S \leqq 0$ implies that $S=T$.

An immediate consequence of Corollary 3.9 is, that a densely defined operator $T$ with $\operatorname{Im} T \leqq 0$ is maximal if and only if it is operator maximal. Hence by Corollary 3.5 a densely defined operator $T$ with $\operatorname{Im} T \leqq 0$ which is operator maximal is necessarily closed.

The concept of operator maximality is due to Phillips [10], who gave the following result. Our proof is based on Theorem 3.8.

Lemma 3.10. Let $T$ be an operator in $\mathfrak{S}$ with $\operatorname{Im} T \leqq 0$. If $T$ is operator maximal and closed, then $\mathfrak{D}(T)$ is dense in $\mathfrak{S}$.

Proof. As in [10] it can be shown that $\Re(T-\lambda)=\mathfrak{F}$ for $\lambda \in C^{+}$. For such $\lambda, C_{\lambda}(T)$ is a contraction with $\mathfrak{D}\left(C_{\lambda}(T)\right)=\mathscr{F}$ and $\nu\left(C_{\lambda}(T)-I\right)=$ $T(0)=\{0\}$. Applying Theorem 3.8 we find $\mathscr{D}(T)=\mathfrak{R}\left(C_{\lambda}(T)-I\right)$ is dense in $\mathfrak{F}$.

CoRollary 3.11. Let $T$ be an operator in $\mathfrak{S}$ with $\operatorname{Im} T \leqq 0$. Then $T$ is maximal if and only if $T$ is operator maximal and closed.

For an example of an operator $T$ in $\mathfrak{S}$ with $\operatorname{Im} T \leqq 0$, which is operator maximal, but not closed (and hence not maximal) we refer to Phillips [10].

THEOREM 3.12. Let $T=T_{s} \oplus T_{\infty}$ be a linear manifold in $\mathfrak{S}^{2}$ with $\operatorname{Im} T \leqq 0(\max )$. Then $T_{s}$ is a densely defined operator in $\mathfrak{S} \ominus T(0)$ with $\operatorname{Im} T_{s} \leqq 0(\max )$. 
Proof. Since $T$ is maximal it follows from Theorem 3.4 that $\Re(T-i)=\mathfrak{S}$ and hence by Proposition 2.2 we have $\Re\left(T_{s}-i\right)=\mathfrak{K} \ominus$ $T(0)$. This implies $\mathfrak{D}\left(C_{i}\left(T_{s}\right)\right)=\mathfrak{S} \ominus T(0)$. From Lemma 2.6 we infer that $\nu\left(C_{\imath}(T)-I\right)=T(0)$. Hence $C_{i}\left(T_{s}\right)$ is the restriction of $C_{\imath}(T)$ to $\mathfrak{S} \ominus \nu\left(C_{i}(T)-I\right)$. An application of Theorem 3.8 completes the proof.

We shall now present analogous results for symmetric linear manifolds $S$ in $\mathfrak{S}^{2}$. A linear manifold $S$ in $\mathscr{S}^{2}$ is symmetric if and only if for some (and hence for all) $\lambda \in \boldsymbol{C}-\boldsymbol{R}$ and for all $\{f, g\} \in S$.

$$
\operatorname{Im}(f, g-\lambda f)=(\operatorname{Im} \lambda)(f, f) .
$$

A consequence of (3.4) is

$$
\|g-\lambda f\| \geqq|\operatorname{Im} \lambda|\|f\|, \lambda \in C-R,
$$

for all $\{f, g\} \in S$.

TheOREM 3.13. Let $S$ be a symmetric linear manifold in $\mathfrak{S}^{2}$. Then

(i) $(S-\lambda)^{-1}$ is an operator, $\lambda \in \boldsymbol{C}-\boldsymbol{R}$,

(ii) $\left\|(S-\lambda)^{-1}\right\| \leqq 1 /|\operatorname{Im} \lambda|, \lambda \in \boldsymbol{C}-\boldsymbol{R}$,

(iii) if $\Re(S-\lambda)$ is closed for some $\lambda \in \boldsymbol{C}-\boldsymbol{R}$, then $S$ is closed. If in addition $S$ is closed, then for all $\lambda \in \boldsymbol{C}-\boldsymbol{R}$

(iv) $\mathfrak{R}(S-\lambda)$ is closed,

(v) $\Re(S-\lambda)=\left(\nu\left(S^{*}-\bar{\lambda}\right)\right)^{\perp}$,

(vi) $\mathfrak{R}\left(S^{*}-\lambda\right)=\mathfrak{S}$.

THEOREM 3.14. Let $S$ be a symmetric linear manifold in $\mathfrak{S}^{2}$. If $\lambda \in \boldsymbol{C}-\boldsymbol{R}$ then $C_{\lambda}(S)$ is an isometric operator.

THEOREM 3.15. Let $V$ be an isometric operator in $\mathfrak{S B}$ defind on $\mathfrak{D}(V)$. If $\lambda \in \boldsymbol{C}-\boldsymbol{R}$ then $F_{\lambda}(V)$ is a symmetric manifold in $\mathfrak{S}_{2}{ }^{2}$.

We shall say that the symmetric linear manifold $S$ in $\mathscr{S}_{2}^{2}$ is maximal if the existence of a symmetric linear manifold $T$ in $\mathscr{S}^{2}$ with $S \subset T$ implies $T=S$.

THEOREM 3.16. Let $S$ be a symmetric linear manifold in $\mathfrak{S}^{2}$.

(i) If $S$ is masimal then $\Re(S-\lambda)=\mathscr{S}$ for all $\lambda \in C^{+}$or for all $\lambda \in \mathbb{C}^{-}$.

(ii) If $\Re(S-\lambda)=\mathfrak{S}$ for some $\lambda \in \mathbb{C}^{+}$or for some $\lambda \in C^{-}$then $S$ is maximal.

Corollary 3.17. Let $S$ be a maximal symmetric linear manifold in $\mathfrak{S}^{2}$. Then $S$ is closed. 
THEOREM 3.18. Let $S$ be a symmetric linear manifold in $\mathfrak{S}^{2}$ and $R(\lambda)=(S-\lambda)^{-1}, \lambda \in C$.

(i) If $S$ is maximal, then for all $\lambda \in C^{+}$or for all $\lambda \in C^{-} R(\lambda)$ exists as a linear operator defined on all of $\mathfrak{S}$ and

$$
\operatorname{Im}(R(\lambda) h, h)=(\operatorname{Im} \lambda)\|R(\lambda) h\|^{2}, h \in \mathfrak{F} .
$$

(ii) If for some $\lambda \in C^{+}$or for some $\lambda \in C^{-} R(\lambda)$ exists as a linear operator defined on all of $\mathfrak{S}$ and satisfies (3.6) then $S$ is maximal.

Operator maximality for symmetric operators is defined in an obvious way. We shall not state explicitly the results corresponding to Lemma 3.10 and Corollary 3.11. The next result was proved by Bennewitz [3] in a different way.

THEOREM 3.19. Let $S=S_{s} \oplus S_{\infty}$ be a maximal symmetric linear manifold in $\mathfrak{S C}^{2}$. Then $S_{s}$ is a densely defined, maximal symmetric operator in $\mathfrak{S} \ominus S(0)$.

Finally we present some results for self-adjoint subspaces in $\mathfrak{S}^{2}$, cf. [2] and [4].

THEOREM 3.20. Let $H$ be a self-adjoint subspace in $\mathfrak{S}^{2}$. Then for all $\lambda \in \boldsymbol{C}-\boldsymbol{R}$
(i) $(H-\lambda)^{-1}$ is an operator,
(ii) $\left\|(H-\lambda)^{-1}\right\| \leqq 1 /|\operatorname{Im} \lambda|$,
(iii) $\mathfrak{R}(H-\lambda)=\mathfrak{S}$.

THEOREM 3.21. Let $H$ be a self-adjoint subspace in $\mathfrak{S}^{2}$. Then for all $\lambda \in \boldsymbol{C}-\boldsymbol{R} C_{\lambda}(H)$ is a unitary operator in $\mathfrak{S}$.

Proof. By Theorem $3.14 C_{\lambda}(H)$ is isometric. Theorem 3.20 shows that $\mathfrak{D}\left(C_{\lambda}(H)\right)=\mathfrak{R}(H-\lambda)$ and $\mathfrak{R}\left(C_{\lambda}(H)\right)=\mathfrak{R}(H-\bar{\lambda})$ are equal to all of $\mathfrak{S}_{2}$. Hence $C_{\lambda}(H)$ is unitary.

THEOREM 3.22. Let $U$ be a unitary operator in $\mathfrak{F}$. For all $\lambda \in \boldsymbol{C}-\boldsymbol{R} F_{\lambda}(U)$ is a self-adjoint subspace in $\mathfrak{S}^{2}$.

Proof. By Theorem $3.15 F_{\lambda}(U)$ is symmetric. We also observe that $\Re\left(F_{\lambda}(U)-\lambda\right)=\mathfrak{R}\left(F_{\lambda}(U)-\bar{\lambda}\right)=\mathfrak{S}$. Hence application of Lemma 2.5 shows that $F_{\lambda}(U)$ is self-adjoint.

THEOREM 3.23. Let $H=H_{s} \oplus H_{\infty}$ be a self-adjoint subspace in $\mathfrak{S}^{2}$. Then $H_{s}$ is a densely defined self-adjoint operator in $\mathfrak{S} \ominus H(0)$. 
4. Various linear manifolds and corresponding operator families. Let $\mathbf{R}(\mathfrak{F})$ denote the class of all functions $R$ from $C-R$ to $\boldsymbol{B}(\mathfrak{K})$ (all bounded linear operators defined on all of $\mathfrak{S}$ ) such that

$$
\begin{gathered}
R(\lambda)^{*}=R(\bar{\lambda}), \lambda \in \boldsymbol{C}-\boldsymbol{R}, \\
R(\lambda)-R(\mu)=(\lambda-\mu) R(\lambda) R(\mu), \lambda, \mu \in \boldsymbol{C}-\boldsymbol{R} .
\end{gathered}
$$

Note that (4.1) and (4.2) imply the inequality

$$
\|R(\lambda)\| \leqq \frac{1}{|\operatorname{Im} \lambda|}, \lambda \in \boldsymbol{C}-\boldsymbol{R}
$$

THEOREM 4.1. (i) Let $R \in \mathbf{R}(\mathfrak{S})$, then the linear manifold $H$ defined by

$$
H=\{\{R(\lambda) h, \lambda R(\lambda) h+h\} / h \in \mathfrak{S}\}, \lambda \in C-R,
$$

is self-adjoint in $\mathfrak{S}^{2}$ and $R(\lambda)=(H-\lambda)^{-1}(\lambda \in \boldsymbol{C}-\boldsymbol{R})$.

(ii) Let $H$ be a self-adjoint subspace in $\mathfrak{S}^{2}$ and let $R(\lambda)=$ $(H-\lambda)^{-1}(\lambda \in \boldsymbol{C}-\boldsymbol{R})$. Then $R \in \mathbf{R}(\mathfrak{K})$ and (4.3) holds.

(iii) If $R \in \mathbf{R}(\mathfrak{S})$ and the self-adjoint subspace $H$ in $\mathfrak{S C}^{2}$ are connected by (4.3) then

$$
\nu(R(\lambda))=H(0), \lambda \in \boldsymbol{C}-\boldsymbol{R} .
$$

Proof. In order to show that $H$ defined by (4.3) does not depend on $\lambda$ we denote the righthand side of (4.3) by $H(\lambda)$. It clearly suffices to show $H(\lambda) \subset H(\mu)$ for $\lambda, \mu \in \boldsymbol{C}-\boldsymbol{R}$. This inclusion holds on account of (4.2) because

$$
\{R(\lambda) h, \lambda R(\lambda) h+h\}=\{R(\mu) k, \mu R(\mu) k+\hbar\}
$$

where for $h \in \mathscr{F}$ the element $k \in \mathfrak{F}$ is defined by $k=h+(\lambda-\mu) R(\lambda) h$. Since $H-\lambda=\left\{\{R(\lambda) h, h\} / h \in \mathfrak{S}_{\mathfrak{c}}\right\}=R(\lambda)^{-1}$, we have

$$
H^{*}-\bar{\lambda}=(H-\lambda)^{*}=\left(R(\lambda)^{-1}\right)^{*}=\left(R(\lambda)^{*}\right)^{-1}=(R(\bar{\lambda}))^{-1}=H-\bar{\lambda},
$$

which is equivalent to $H=H^{*}$. This proves (i). If $H=H^{*}$ and $R(\lambda)=(H-\lambda)^{-1}$ then $R(\lambda) \in \boldsymbol{B}(\mathfrak{K})$ for $\lambda \in \boldsymbol{C}-\boldsymbol{R}$ by Theorem 3.20. Thus (4.2) follows from Lemma 2.4, and (4.1) follows by direct verification. This proves (ii). In order to prove (iii) we note

$$
f \in \nu(R(\lambda)) \Longleftrightarrow\{f, 0\} \in R(\lambda) \Longleftrightarrow\{0, f\} \in H \Longleftrightarrow f \in H(0) .
$$

Let $\mathbf{E}(\mathfrak{S})$ denote the class of all functions $E$ from $\boldsymbol{R}$ to $\boldsymbol{B}(\mathfrak{S})$ such that

$$
E(t)^{*}=E(t), t \in R,
$$




$$
\begin{gathered}
E(t) E(s)=E(\min (t, s)), t, s \in R, \\
\lim _{t \rightarrow-\infty} E(t)=0 \quad(\text { strong }) .
\end{gathered}
$$

We assume $E$ to be right continuous (strong). Note that $E(\infty)$ exists (strong limit) and is an orthogonal projection, cf. [1].

THEOREM 4.2. (i) Let $E \in \mathbf{E}(\mathfrak{S})$, then the function $R$ defined by

$$
R(\lambda)=\int_{\boldsymbol{R}} \frac{1}{t-\lambda} d E(t), \lambda \in \boldsymbol{C}-\boldsymbol{R},
$$

belongs to $\mathbf{R}(\mathfrak{S})$.

(ii) Let $R \in \mathbb{R}(\mathfrak{S})$ then there exists a function $E \in \mathbf{E}(\mathfrak{S})$ such that (4.7) holds.

(iii) If $E \in \mathbf{E}(\mathfrak{S})$ and $R \in \mathbf{R}(\mathfrak{S})$ are connected by (4.7) then

$$
\nu(E(\infty))=\nu(R(\lambda)), \lambda \in \boldsymbol{C}-\boldsymbol{R} .
$$

The proof of (i) is straightforward, while (ii) depends upon a representation theorem of Nevanlinna (cf. [1]). For details we refer to $[1],[6]$, and [11].

We have now seen that to every self-adjoint subspace $H$ in $\mathfrak{S}^{2}$ there exists a function $E \in \mathbf{E}(\mathfrak{F})$ such that

$$
(H-i)^{-1}=\int_{-\infty}^{\infty} \frac{1}{t-i} d E(t) .
$$

On the other hand, since $C_{i}(H)$ is a unitary operator in $\mathscr{S}$ there exists a spectral family $F$ such that

$$
C_{\imath}(H)=\int_{0}^{2 \pi} e^{-i s} d F(s) .
$$

THEOREM 4.3. $E(t)=F(s)$ with $t=-\operatorname{cotg} s / 2$.

Proof. If $\lambda \in C-\mathbb{R}$ then $H=F_{i}\left(C_{\imath}(H)\right)$ implies $R(\lambda)=$ $\left(C_{2}(H)-I\right)\left[(i-\lambda) C_{2}(H)+(\lambda+i)\right]^{-1}$. Hence

$$
\int_{-\infty}^{\infty} \frac{1}{t-\lambda} d E(t)=\int_{0}^{2 \pi} \frac{e^{-i s}-1}{(i-\lambda) e^{-i s}+(\lambda+i)} d F(s), \lambda \in C-R .
$$

Setting $t=-\operatorname{cotg} s / 2$ we obtain the desired result.

The above results show that a self-adjoint subspace $H$ in $\mathfrak{S}^{2}$ determines, and is determined by, each of the functions $R \in \mathbb{R}(\mathfrak{S})$ and $E \in \mathbb{E}(\mathfrak{S})$. Let $R_{s}(\lambda)$ and $E_{\mathrm{s}}(t)$ be the restrictions of $R(\lambda)$ and $E(t)$ to $\mathfrak{g} \ominus H(0)$ respectively. Theorem 3.23 shows the decomposition $H=$ $H_{s} \oplus H_{\infty}$ where $H_{s}$ is a densely defined self-adjoint operator in $\mathfrak{E} \ominus$ 
$H(0)$. We then have $R_{s}(\lambda)=\left(H_{s}-\lambda\right)^{-1}$ and

$$
R_{s}(\lambda)=\int_{R} \frac{1}{t-\lambda} d E_{s}(t)
$$

with $E_{s}(\infty)=I$ in $\mathscr{S} \ominus H(0)$.

We will now present similar results for function classes extending $\mathbf{R}(\mathfrak{S})$ and $\mathbf{E}(\mathfrak{S})$. In particular the self-adjoint subspace in Theorem 4.1 will be replaced by an analytic family of subspaces.

Let $\boldsymbol{R}(\mathfrak{S})$ denote the class of all functions $R$ from $\boldsymbol{C}-\boldsymbol{R}$ to $\boldsymbol{B}(\mathfrak{S})$ such that

$$
R(\lambda)^{*}=R(\bar{\lambda}), \lambda \in \boldsymbol{C}-\boldsymbol{R},
$$

$R$ is holomorphic on $\boldsymbol{C}-\boldsymbol{R}$,

$$
\|R(\lambda) h\|^{2} \leqq \frac{\operatorname{Im}(R(\lambda) h, h)}{\operatorname{Im} \lambda}, h \in \mathfrak{S}, \lambda \in C-\boldsymbol{R} .
$$

We remark that $\mathbf{R}(\mathfrak{S}) \subset \boldsymbol{R}(\mathfrak{S})$.

LEMMA 4.4. Let $R \in R(\mathfrak{S})$, then $\nu(R(\lambda))=\nu(R(\mu))$ for all $\lambda, \mu \in C-$ R.

Proof. From (4.10) it follows that $\nu(R(\lambda))=\{h \in \mathfrak{S} /(R(\lambda) h, h)=0\}$ for $\lambda \in \boldsymbol{C}-\boldsymbol{R}$. The condition (4.8) implies

$$
\nu(R(\bar{\lambda}))=\{h \in \mathfrak{S} /(R(\bar{\lambda}) h, h)=0\}=\{h \in \mathfrak{S} /(h, R(\lambda) h)=0\}=\nu(R(\lambda)) .
$$

So it suffices to prove the lemma for $\lambda, \mu \in C^{+}$. For $h \in \mathfrak{K}$ we define $\phi(\lambda)=(R(\lambda) h, h)$, then by (4.9) $\phi$ is analytic in $C^{+}$and by (4.10) $\operatorname{Im} \phi(\lambda) \geqq 0$ for $\lambda \in C^{+}$. Suppose $\phi\left(\lambda_{0}\right)=0$ for $\lambda_{0} \in \boldsymbol{C}^{+}$. Choose $r>0$ such that $\left\{\lambda /\left|\lambda-\lambda_{0}\right| \leqq r\right\} \subset C^{+}$. For all $\rho$ with $0 \leqq \rho<r$ we have

$$
0=\dot{\phi}\left(\lambda_{0}\right)=\frac{1}{2 \pi i} \int_{\left|z-\lambda_{0}\right|=\rho} \frac{\dot{\phi}(z)}{z-\lambda_{0}} d z=\frac{1}{2 \pi} \int_{0}^{2 \pi} \dot{\phi}\left(\lambda_{0}+e^{i \theta}\right) d \theta .
$$

Taking imaginary parts we obtain $\operatorname{Im} \phi\left(\lambda_{0}+\rho e^{i \theta}\right)=0$ for all $\rho$ with $0 \leqq \rho<r$ and $\theta$ with $0 \leqq \theta \leqq 2 \pi$. This shows that $\phi(\lambda)=0$ for all $\lambda \in \boldsymbol{C}^{+}$and hence for all $\lambda \in \boldsymbol{C}-\boldsymbol{R}$. This completes the proof.

In order to prove a result corresponding to Theorem 4.1 we introduce the class $\boldsymbol{T}(\mathfrak{K})$ of functions $T$ from $\boldsymbol{C}-\boldsymbol{R}$ into the linear manifolds on $\mathfrak{S}^{2}$ such that

$$
\begin{gathered}
\operatorname{Im} T(\lambda) \leqq 0(\max ), \lambda \in C^{+}, \\
T(\bar{\lambda})=T(\lambda)^{*}, \lambda \in C^{+},
\end{gathered}
$$

(4.13) $C_{\mu}(T(\lambda))$ defines a holomorphic function in $\lambda \in C^{+}$for some (and hence for all) $\mu \in C^{+}$. 
Note that if $H$ is a self-adjoint subspace in $\mathfrak{S}^{2}$ and if we set $T(\lambda)=H$ for all $\lambda \in \boldsymbol{C}-\boldsymbol{R}$, then $T \in \boldsymbol{T}(\mathfrak{S})$.

THEOREM 4.5. (i) Let $R \in \boldsymbol{R}(\mathfrak{S})$, then the function $T$ defined by

$$
T(\lambda)=\{\{R(\lambda) h, \lambda R(\lambda) h+h\} / h \in \mathfrak{S}\}, \lambda \in \boldsymbol{C}-\boldsymbol{R},
$$

belongs to $\boldsymbol{T}(\mathfrak{S})$ and $R(\lambda)=(T(\lambda)-\lambda)^{-1}(\lambda \in \boldsymbol{C}-\boldsymbol{R})$.

(ii) Let $T \in T(\mathfrak{S})$ and let $R(\lambda)=(T(\lambda)-\lambda)^{-1}(\lambda \in C-R)$. Then $R \in \boldsymbol{R}(\mathfrak{S})$ and (4.14) holds.

(iii) If $R \in \boldsymbol{R}(\mathfrak{S})$ and $T \in \boldsymbol{T}(\mathfrak{S})$ are connected by (4.14), then

$$
\nu(R(\lambda))=T(\lambda)(0), \lambda \in \boldsymbol{C}-\boldsymbol{R} .
$$

Proof. Let $T(\lambda)$ be defined by (4.14), then it follows from (4.10) for all $\lambda \in C^{+}$that

$$
\operatorname{Im}(R(\lambda) h, \lambda R(\lambda) h+h) \geqq 0, h \in \mathfrak{S},
$$

which shows $\operatorname{Im} T(\lambda) \leqq 0$. The assertion (4.12) follows directly from $T(\lambda)-\lambda=R(\lambda)^{-1}$. Since $\mathfrak{R}(T(\lambda)-\lambda)=\mathfrak{D}(R(\lambda))=\mathfrak{F}, T(\lambda)$ is maximal by Theorem 3.4. For $\lambda, \mu \in C^{+}$we consider

$$
C_{\lambda, \mu}(T(\lambda))=\{\{g-\lambda f, g-\mu f\} /\{f, g\} \in T(\lambda)\} .
$$

It is clear that $C_{\lambda, \mu}(T(\lambda))$ is an injective operator, mapping all of $\mathfrak{S}$ onto itself, and also that

$$
C_{\lambda, \mu}(T(\lambda))=I+(\lambda-\mu) R(\lambda) .
$$

Therefore the inverse of $C_{\lambda, \mu}(T(\lambda))$

$$
\left(C_{\lambda, \mu}(T(\lambda))\right)^{-1}=[I+(\lambda-\mu) R(\lambda)]^{-1}=C_{\mu, \lambda}(T(\lambda))
$$

is a bounded operator defined on all of $\mathscr{F}$ and holomorphic (in $\lambda$ ) in $C^{+}$. It is straightforward that $(T(\lambda)-\mu)^{-1}$ is a bounded operator defined on all of $\mathfrak{H}$ and

$$
(T(\lambda)-\mu)^{-1}=R(\lambda) C_{\mu, \lambda}(T(\lambda))
$$

and also that

$$
C_{\mu}(T(\lambda))=I+(\mu-\bar{\mu})(T(\lambda)-\mu)^{-1} .
$$

Taking (4.15), (4.16), and (4.17) together we find

$$
C_{\mu}(T(\lambda))=I+(\mu-\bar{\mu}) R(\lambda)[I+(\lambda-\mu) R(\lambda)]^{-1} .
$$

Hence $C_{\mu}(T(\lambda))$ defines a holomorphic function in $\lambda \in C^{+}$(for $\mu \in C^{+}$). This proves (i). Now we let $T \in \boldsymbol{T}(\mathfrak{S})$ and $R(\lambda)=(T(\lambda)-\lambda)^{-1}$. The assertions (4.8) and (4.10) follow directly from (4.11) and (4.12). Using 
$T(\lambda)=F_{\mu}\left(C_{\mu}(T(\lambda))\right)$ we obtain

$$
T(\lambda)=\left\{\left\{C_{\mu}(T(\lambda)-I) h,\left(\mu C_{\mu}(T(\lambda))-\bar{\mu}\right) h\right\} / h \in \mathfrak{S}_{\mathfrak{E}}\right\}
$$

or

$$
R(\lambda)=\left\{\left\{(\mu-\lambda) C_{\mu}(T(\lambda)) h+(\lambda-\bar{\mu}) h,\left(C_{\mu}(T(\lambda))-I\right) h\right\} / h \in \mathfrak{S}_{\mathcal{E}}\right\}
$$

which shows

$$
R(\lambda)=\left(C_{\mu}(T(\lambda))-I\right)\left[(\mu-\lambda) C_{\mu}(T(\lambda))+(\lambda-\bar{\mu})\right]^{-1} .
$$

For $\lambda, ", \in C^{+}$we have

$$
|\lambda-\bar{\mu}|>|\lambda-\mu|
$$

and this shows that for $\lambda=\mu$ and for $\lambda \neq \mu$ the linear manifold

$$
\left[(\mu-\lambda) C_{\mu}(T(\lambda))+(\lambda-\bar{\mu})\right]^{-1}
$$

is a bounded operator defined on all of $\mathfrak{S}$. This together with (4.18) implies that the function $R$ is holomorphic in $C^{+}$. From (4.8) we obtain the holomorphy of $R$ in all of $\boldsymbol{C}-\boldsymbol{R}$. This proves (ii). The proof of (iii) is straightforward.

Lemma 4.4 and Theorem 4.5 show that for $T \in \boldsymbol{T}(\mathfrak{S})$ the space $T(\lambda)(0)$ does not depend on $\lambda \in \boldsymbol{C}-\boldsymbol{R}$. We shall therefore use the notation $T(0)$ to indicate the space $T(\lambda)(0)$.

Let $\boldsymbol{E}(\mathfrak{S})$ denote the class of all functions $E$ from $R$ to $\boldsymbol{B}(\mathfrak{S})$ such that

$$
\begin{gathered}
0 \leqq(E(t) h, h) \leqq(E(s) h, h) \leqq(h, h), t<s, h \in \tilde{\mathfrak{F}}, \\
\lim _{t \rightarrow-\infty} E(t)=0 \quad \text { (strong). }
\end{gathered}
$$

We assume $E$ to be right continuous (strong). Note that $E(\infty)$ exists (strong limit) and $E(\infty) \leqq I$, cf. [1]. We remark $\mathrm{E}(\mathfrak{S}) \subset E(\mathfrak{S})$.

THEOREM 4.6. (i) Let $E \in \boldsymbol{E}(\mathfrak{S})$, then the function $R$ defined by (4.7) belongs to $R(\mathfrak{S})$.

(ii) Let $R \in \boldsymbol{R}(\mathfrak{S})$ then there exists a function $E \in \boldsymbol{E}(\mathfrak{S})$ such that (4.7) holds.

(iii) If $E \in E(\mathfrak{S})$ and $R \in R(\mathfrak{S})$ and connected by (4.7) then

$$
\nu(E(\infty))=\nu(R(\lambda)), \lambda \in \boldsymbol{C}-\boldsymbol{R} .
$$

For the proof we refer to [6]. Analogous to the decomposition Theorems 3.12 and 3.23 we now state a decomposition theorem for $T \in \boldsymbol{T}(\mathfrak{S})$. By $\boldsymbol{T}_{s}(\mathfrak{F})$ we denote the class of all functions $T$ from $\boldsymbol{C}-\boldsymbol{R}$ into the densely defined linear operators in $\mathfrak{S}$ such that (4.11), (4.12), and (4.13) hold. 
THEOREM 4.7. Let $T \in \boldsymbol{T}(\mathfrak{S})$ and let $T_{s}$ denote the function defined on $\boldsymbol{C}-\boldsymbol{R}$ with values $T(\lambda)_{s}$ given by the decomposition

$$
T(\lambda)=T(\lambda)_{s} \oplus T(\lambda)_{\infty}, \lambda \in C-R .
$$

Then $T_{s} \in T_{s}(\mathfrak{S} \ominus T(0))$.

Proof. It follows from Theorems 3.12 and 4.5 that $T(\lambda)_{s}$ is a densely defined linear operator in $\mathscr{S} \ominus T(0)$ with $\operatorname{Im} T(\lambda)_{s} \leqq 0$ (max). $C_{\mu}\left((T(\lambda))_{s}\right)$ is a restriction of $C_{\mu}((T(\lambda)))$ to $\mathfrak{S} \ominus T(0)$; hence for $\mu \in C^{+}$ it defines a holomorphic function (of $\lambda$ ) in $C^{+}$. Restricting the relation (4.12) to $\mathfrak{S} \ominus T(0)$ we find

$$
T(\bar{\lambda})_{s}=\left(T(\lambda)_{s}\right)^{*}, \lambda \in C^{+}
$$

where the adjoint is now taken in $\mathscr{\mathcal { C }} \ominus T(0)$. This proves the theorem.

We remark that a function $T \in \boldsymbol{T}(\mathfrak{S})$ determines, and is determined by, each of the functions $R \in R\left(\mathscr{F}_{\mathcal{C}}\right)$ and $E \in E\left(\mathfrak{F}_{\mathcal{C}}\right)$. Let $R_{s}(\lambda)$ and $E_{s}(t)$ be the restrictions of $R(\lambda)$ and $E(t)$ to $\mathscr{S} \ominus T(0)$ respectively. From Theorem 4.7 we have the decomposition $T(\lambda)=T(\lambda)_{s} \oplus T(\lambda)_{\infty}$ with $T(\lambda)_{s}$ determining a function $T_{s} \in T_{s}(\mathfrak{S} \ominus T(0))$. We find the relations $R_{s}(\lambda)=\left(T(\lambda)_{s}-\lambda\right)^{-1}$ and

$$
R_{s}(\lambda)=\int_{R} \frac{1}{t-\lambda} d E_{s}(t)
$$

It does not necessarily follow that $E_{s}(\infty)=I$ in $\mathfrak{S} \ominus T(0)$.

Let $\mathfrak{S}$ and $\mathscr{K}$ be Hilbert spaces suct that $\mathscr{K} \subset \Re$ and let $P$ be the orthogonal projection of $\Omega$ onto $\mathfrak{S}$. If $E \in \mathbf{E}(\Omega)$ and $E(t)=\left.P E(t)\right|_{\tilde{n}}$, then $\boldsymbol{E} \in \boldsymbol{E}(\mathfrak{S})$. Also $E(\infty)=I$ implies $E(\infty)=I$, and $E(\Delta)=0$ implies $E(\Delta)=0$. Here we use the notation $E(\Delta)=E(b)-E(a)$ for the interval $\Delta=(a, b]$, correspondingly for $\boldsymbol{E}(\Delta)$. We shall now state a converse result. It is a generalization of a theorem of Naimark [8], due to McKelvey [6].

THEOREM 4.8. Let $\boldsymbol{E} \in \boldsymbol{E}(\mathfrak{S})$. Then there exists a Hilbert space $\Re$ such that $\mathfrak{K} \subset \Re$ and a function $E \in \mathbf{E}(\Re)$ such that

(i) $\boldsymbol{E}(t)=\left.P E(t)\right|_{\mathfrak{n}}$, where $P$ is the projection of $\Re$ onto $\mathfrak{S}$,

(ii) $\Re$ is spanned by $\mathfrak{S}$ and $\{E(t) h h \in \mathfrak{S}, t \in \boldsymbol{R}\}$,

(iii) $E(\infty)=I$ if and only if $E(\infty)=I ; E(\Delta)=0$ if and only if $\boldsymbol{E}(\Delta)=0$.

5. Unitary extensions of isometric operators. Before we consider self-adjoint extensions of symmetric subspaces we shall present the corresponding theory for unitary extensions of closed isometric 
operators. In this section all isometric operators are supposed to be closed. For reference we state the following simple result.

THEOREM 5.1. Let $V$ be an isometric operator in $\mathfrak{S}$, and let $V^{\prime}$ be an isometric extension of $V$ in $\mathfrak{S}$. Then

$$
V^{\prime}=V \oplus W
$$

where $W$ is an isometric operator mapping a subspace of $\mathfrak{S} \ominus \mathfrak{D}(V)$ into $\mathfrak{S} \ominus \Re(V)$. Conversely let $V$ and $W$ be as above, then $V^{\prime}$ defined by (5.1) is an isometric extension of $V$ in $\mathfrak{S}$.

For a given isometric operator $V$ in $\mathscr{S}$ there do not necessarily exist unitary extensions on $\mathfrak{S}$. But if we extend $V$ in the trivial way to a contraction operator $T$ with $\mathfrak{D}(T)=\mathfrak{F}_{\mathfrak{C}}$, then as Halmos showed (cf. [13]) there exists a Hilbert space $\Re$ with $\mathfrak{S} \subset \Re$ and a unitary operator $U$ on $\Omega$ such that $T \subset P U$, where $P$ is the orthogonal projection of $\Re$ onto $\mathfrak{F}$. Since $V$ is isometric it follows that $V \subset U$.

The following theorem shows how all unitary extensions of a given isometric operator are to be constructed.

THEOREM 5.2. Let $V_{1}$ be an isometric operator in $\mathfrak{S}_{1}$. Let $V_{1} \subset$ $U$ where $U$ is a unitary operator on $\Re=\mathfrak{S}_{1} \oplus \mathfrak{\mathcal { S }}_{2}$. Let $V_{2}$ be the restriction of $U$ to the subspace $\left\{f \in \mathfrak{F}_{2} / U f \in \mathfrak{S}_{2}\right\}$ of $\mathfrak{S}_{2}$. Then $V_{2}$ is an isometric operator in $\mathfrak{F}_{2}$ such that

$$
\begin{aligned}
& \operatorname{dim}\left(\mathfrak{S}_{2} \ominus \mathfrak{R}\left(V_{2}\right)\right) \leqq \operatorname{dim}\left(\mathfrak{S}_{1} \ominus \mathfrak{D}\left(V_{1}\right)\right) \\
& \operatorname{dim}\left(\mathfrak{S}_{1} \ominus \mathfrak{D}\left(V_{1}\right)\right)+\operatorname{dim}\left(\mathscr{S}_{2} \ominus \mathfrak{D}\left(V_{2}\right)\right) \\
& =\operatorname{dim}\left(\mathfrak{S}_{1} \ominus \mathfrak{R}\left(V_{1}\right)\right)+\operatorname{dim}\left(\mathfrak{S}_{2} \ominus \mathfrak{R}\left(V_{2}\right)\right),
\end{aligned}
$$

and $U$ can be written in the form

$$
U=V_{1} \oplus V_{2} \oplus W
$$

where $W$ is an isometric operator in $\mathfrak{S}_{1} \oplus \mathfrak{S}_{2}$ such that

$$
\begin{gathered}
\mathfrak{D}(W)=\left(\mathfrak{S}_{1} \ominus \mathfrak{P}\left(V_{1}\right)\right) \oplus\left(\mathfrak{S}_{2} \ominus \mathfrak{D}\left(V_{2}\right)\right), \\
\mathfrak{R}(W)=\left(\mathfrak{S}_{1} \ominus \mathfrak{R}\left(V_{1}\right)\right) \oplus\left(\mathfrak{S}_{2} \ominus \mathfrak{R}\left(V_{2}\right)\right), \\
P_{1} W^{-1} \text { maps } \mathfrak{S}_{2} \ominus \Re\left(V_{2}\right) \text { one to one into } \mathfrak{S}_{1} \ominus \mathfrak{D}\left(V_{1}\right)
\end{gathered}
$$

or equivalently

$$
P_{2} W \text { maps } \mathfrak{S}_{1} \ominus \mathfrak{D}\left(V_{1}\right) \text { onto } \mathfrak{S}_{2} \ominus \mathfrak{R}\left(V_{2}\right),
$$

where $P_{j}(j=1,2)$ is the orthogonal projection from $\mathfrak{F}_{1} \oplus \mathfrak{S}_{2}$ onto $\mathfrak{S}_{j}$. 
Conversely, suppose that for a given isometric operator $V_{1}$ in $\mathfrak{S}_{1}$ there are a Hilbert space $\mathfrak{S}_{2}$, an isometric operator $V_{2}$ in $\mathfrak{F}_{2}$ satisfying (5.2) and (5.3) and an isometric operator $W$ in $\Omega=\mathfrak{S}_{1} \oplus \mathfrak{S}_{2}$ satisfying (5.5) and (5.6). Then $U$ defined by (5.4) is unitary on $\Re$ and $V_{2}$ has the property that $\mathfrak{D}\left(V_{2}\right)=\left\{f \in \mathfrak{F}_{2} / U f \in \mathfrak{S}_{2}\right\}$.

Proof. Define $W$ as the restriction of $U$ to $\mathfrak{D}(W)$ given in (5.5). It is clear that $V_{2}$ and $W$ are isometric. Since $U$ is unitary and maps $\mathfrak{D}\left(V_{1}\right) \oplus \mathfrak{D}\left(V_{2}\right)$ onto $\mathfrak{R}\left(V_{1}\right) \oplus \mathfrak{R}\left(V_{2}\right), U$ and hence $W$ maps $\Re \ominus$ $\left(\mathfrak{D}\left(V_{1}\right) \oplus \mathfrak{D}\left(V_{2}\right)\right)=\mathfrak{D}(W)$ onto $\Re \ominus\left(\Re\left(V_{1}\right) \oplus \mathfrak{R}\left(V_{2}\right)\right)=\mathfrak{R}(W)$. Hence (5.5) holds and thus (5.3) holds also. The decomposition (5.4) of $U$ is now evident. Next we prove (5.6) from which (5.2) immediately follows. Suppose that for some $u \in \mathscr{F}_{2} \ominus \Re\left(V_{2}\right) P_{1} W^{-1} u=0$. Then $y=W^{-1} u \in \mathscr{F}_{2} \ominus \mathfrak{D}\left(V_{2}\right)$. Hence $y$ and $U y=W y=u$ belong to $\mathfrak{F}_{2}$. By definition of $\mathfrak{D}\left(V_{2}\right), y \in \mathfrak{D}\left(V_{2}\right) \cap\left(\mathscr{S}_{2} \ominus \mathfrak{D}\left(V_{2}\right)\right)=\{0\}$. Hence $u=0$. This proves (5.6). If $u \in \mathfrak{S}_{2} \ominus \Re\left(V_{2}\right)$ and $y \in \mathfrak{S}_{1} \ominus \mathfrak{D}\left(V_{1}\right)$, then

$$
\left(P_{1} W^{-1} u, y\right)=\left(u, P_{2} W y\right) \text {. }
$$

If $P_{1} W^{-1} u=0$ then the lefthand side and so also the righthand side of (5.8) equals zero for all $y \in \mathfrak{F}_{1} \ominus \mathfrak{D}\left(V_{1}\right)$. Hence (5.7) implies (5.6). If $\left(u, P_{2} W y\right)=0$ for all $y \in \mathfrak{S}_{1} \ominus \mathfrak{D}\left(V_{1}\right)$ then by (5.8) $P_{1} W^{-1} u=0$. Hence (5.6) implies (5.7).

We now prove the second part of the theorem. The operator $U$ defined by (5.4) maps all of $\Omega$ isometrically onto $\Re$ and hence is

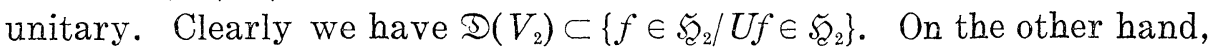
suppose that $f$ and $U f$ belong to $\mathfrak{S}_{2}$. Then $f=f_{1}+f_{2}, f_{1} \in \mathscr{K}_{2} \ominus \mathfrak{D}\left(V_{2}\right)$ and $f_{2} \in \mathfrak{D}\left(V_{2}\right)$ and hence $f_{1}$ and $W f_{1}=U f-V_{2} f_{2}$ belong to $\mathfrak{S}_{2}$. So $P_{1} W^{-1} W f_{1}=P_{1} f_{1}=0$ and hence by (5.6) this implies $W f_{1}=0$. Hence $f_{1}=0$, i.e., $f \in \mathfrak{D}\left(V_{2}\right)$. Thus $V_{2}$ bas the stated property.

COROLlaRy 5.3. Let $V$ be an isometric operator in $\mathfrak{K}$. Then there exists a unitary extension of $V$ on $\mathfrak{S E}$ if and only if

$$
\operatorname{dim}(\mathfrak{S} \ominus \mathfrak{R}(V))=\operatorname{dim}(\mathfrak{S} \ominus \mathfrak{D}(V)) .
$$

If (5.9) holds then all unitary extensions $U$ of $V$ on $\mathfrak{B}$ are of the form $U=V \oplus W$, where $W$ is an isometry from $\mathfrak{S} \ominus \mathfrak{D}(V)$ onto $\mathfrak{S} \ominus \Re(V)$ and conversely each $W$ with this property determines a unitary extension $U$ of $V$ by $U=V \oplus W$.

Let $V$ be an isometric operator in $\mathfrak{S}$ and let $U$ be a unitary extension of $V$ on $\Re, \mathfrak{S} \subset \Omega . \quad U$ is called a minimal extension if

$$
\Re=\overline{\left\langle U^{n} f / f \in \mathscr{S}, n=0, \pm 1, \pm 2, \cdots\right\rangle} .
$$


$(<>$ denotes "linear space spanned by".) From the representation

$$
U=\int_{0}^{2 \pi} e^{-i s} d F(s)
$$

where $F$ is the spectral family associated with $U$ it follows that (5.10) holds if and only if

$$
\Re=\overline{\langle F(s) f \mid f \in \mathfrak{S}, s \in[0,2 \pi]\rangle} .
$$

Let $U$ be a unitary extension of $V$ on $\Omega$. Define $\Re_{1}$ as the closure of the linear subspace of $\Re$ spanned by $\left\{U^{n} f / f \in \mathfrak{F}, n=0, \pm 1, \pm 2, \cdots\right\}$ and let $U_{1}$ be the restriction of $U$ to $\Re_{1}$. Then $U_{1}$ is a minimal unitary extension of $V$ on $\Re_{1}$ and $U_{1} \subset U$.

THEOREM 5.4. Let $V$ be an isometric operator in $\mathfrak{S g}$. For $j=$ 1,2 let $U_{j}$ be a minimal unitary extension of $V$ in $\Re_{j}$ such that

$$
P_{1} U_{1}^{n}=P_{2} U_{2}^{n} \text { on } \mathfrak{F}, \quad n=0,1,2, \cdots
$$

where $P_{j}$ is the orthogonal projection from $\mathfrak{R}_{j}$ on $\mathfrak{S}$. Then there exists an isomorphism $\Phi$ from $\Re_{1}$ onto $\Omega_{2}$ such that $\Phi f=f$ for all $f \in \mathfrak{S}$ and $U_{1}=\Phi^{-1} U_{2} \Phi$.

Proof. Clearly (5.12) also holds for $n=-1,-2, \cdots$. For $f, g \in \mathfrak{S}$ and $n$ and $m$ integers we have

$$
\left(U_{1}^{n} f, U_{1}^{m} g\right)=\left(P_{1} U_{1}^{n-m} f, g\right)=\left(P_{2} U_{2}^{n-m} f, g\right)=\left(U_{2}^{n} f, U_{2}^{m} g\right) .
$$

From this and the minimality of $U_{j}(j=1,2)$ it follows that the continuous continuation $\Phi$ (to all of $\Omega_{1}$ ) of $\Phi^{\prime}$ defined by

$$
\Phi^{\prime}\left(\sum_{n=-k}^{k} U_{1}^{n} f_{n}\right)=\sum_{n=-k}^{k} U_{2}^{n} f_{n}, \quad k=0,1,2, \cdots, f_{n} \in \mathfrak{S}_{c},
$$

maps $\Re_{1}$ isomorphically onto $\Re_{2}$ and has the properties mentioned in the theorem.

We note that (5.12) is equivalent to

$$
P_{1} F_{1}(s)=P_{2} F_{2}(s) \text { on } \mathfrak{S}, s \in[0,2 \pi],
$$

where $F_{j}$ is the spectral family associated with $U_{j}$ on $\Re_{j}(j=1,2)$ by (5.11).

6. Self-adjoint extensions of symmetric subspaces. For a linear manifold $S$ in $\mathscr{S}^{2}$ we define the "deficiency space" $M_{\lambda}(S)$ or $M_{\lambda}$ by

$$
M_{\lambda}=\left\{\{h, k\} \in S^{*} / k=\lambda h\right\}, \lambda \in C .
$$

Note that $M_{\lambda}$ is a linear operator and for $\lambda \in \boldsymbol{C}-\boldsymbol{R}$ 


$$
\mathfrak{D}\left(M_{\lambda}\right)=(\Re(S-\bar{\lambda}))^{\perp} .
$$

TheOREM 6.1. Let $S$ be a symmetric subspace in $\mathrm{Sg}^{2}$. Then

$$
S^{*}=S+M_{\lambda}+M_{\bar{\lambda}}, \lambda \in C-R \text {. }
$$

For $\lambda \in\{i,-i\}$ the direct sum in (6.3) is orthogonal:

$$
S^{*}=S \oplus M_{\lambda} \oplus M_{\bar{\lambda}}, \lambda \in\{i,-i\} .
$$

Furthermore,

$$
\begin{aligned}
& \operatorname{dim} M_{\lambda}=\operatorname{dim} M_{i}, \lambda \in C^{+}, \\
& \operatorname{dim} M_{\lambda}=\operatorname{dim} M_{-i}, \lambda \in C^{-} .
\end{aligned}
$$

The proof of this theorem can be given along the same lines as the proof of the corresponding theorem for symmetric operators (cf. [9]). For $\lambda \in\{i,-i\}$ (6.3) has been proved by Coddington [4].

From the previous section we deduce the following extension theorems, which have been proved in a different manner for the case $\lambda \in\{i,-i\}$ by Coddington [4].

THEOREM 6.2. Let $S$ be a symmetric subspace in $\mathscr{S C}^{2}$. Let $S^{\prime}$ be a symmetric subspace extension of $S$ in $\mathfrak{S}^{2}$. Then for fixed $\lambda \in \boldsymbol{C}-\boldsymbol{R}$

$$
S^{\prime}=S+(I-V) M,
$$

where $V$ is an isometry, mapping a subspace $M$ of $M$; into $M_{\bar{\lambda}}$. Conversely, let $S$ and $V$ be as above then $S^{\prime}$ defined by (6.4) is a symmetric subspace extension of $S$ in $\mathfrak{S}^{2}$. For $\lambda \in\{i,-i\}$ the direct sum in (6.4) is orthogonal.

Proof. Let $V=C_{\lambda}(S), V^{\prime}=C_{\lambda}\left(S^{\prime}\right)$, then $V$ and $V^{\prime}$ are isometries in $\mathfrak{S}$. By Theorem 5.1 there exists an isometric operator $W$ mapping a subspace of $\mathfrak{S C} \ominus \mathfrak{D}(V)=\mathfrak{R}(S-\lambda)^{\perp}=\mathfrak{D}\left(M_{\bar{\lambda}}\right)$ (cf. (6.2)) into a subspace $M_{1}$ of $\mathfrak{S} \ominus \mathfrak{R}(V)=\mathfrak{R}(S-\bar{\lambda})^{\perp}=\mathfrak{D}\left(M_{,}\right)$such that (5.1) holds. Define the subspace $M$ of $M_{\lambda}$ by

$$
M=\left\{\{h, \lambda h\} / h \in M_{1}\right\}
$$

and the isometry $V$ of $M$ into $M_{\bar{\lambda}}$ by

$$
V\{h, \lambda h\}=\left\{W^{-1} h, \bar{\lambda} W^{-1} h\right\} .
$$

From (5.1) and Lemma 2.6 it follows that

$$
\begin{aligned}
S^{\prime} & =F_{\lambda} C_{\lambda}\left(S^{\prime}\right)=F_{\lambda}\left(C_{\lambda}(S) \oplus W\right) \\
& =S+F_{\lambda} W=S+(I-V) M .
\end{aligned}
$$


For $\lambda \in\{i,-i\}$ the direct sums are orthogonal by Lemma 2.7. By defining $W$ by means of (6.5) one can easily deduce the second part of the theorem from the second part of the Theorem 5.1.

From the remarks following Theorem 5.1 one can deduce by using the Cayley transform that each symmetric subspace in $\mathfrak{S}_{2}^{2}$ can be extended to a self-adjoint subspace in $\Re^{2}$ for some Hilbert space $\Re \supset \mathfrak{F}$.

Let $S_{j}$ be a symmetric subspace in $\mathfrak{F}_{j}, j=1,2$. Then $S_{1} \oplus S_{2}$ is a symmetric subspace in $\mathfrak{S}_{1} \oplus \mathfrak{S}_{2}$. Furthermore, let $M_{\lambda}, M_{\lambda}^{1}$, and $M_{\lambda}^{2}$ be defind for $S_{1} \oplus S_{2}$ in $\left(\mathfrak{S}_{1} \oplus \mathfrak{S}_{2}\right)^{2}, S_{1}$ in $\mathfrak{S}_{1}^{2}$ and $S_{2}$ in $\mathfrak{S}_{2}^{2}$ by (6.1), $\lambda \in \boldsymbol{C}-\boldsymbol{R}$. Then

$$
M_{\lambda}=M_{\lambda}^{1} \oplus M_{\lambda}^{2}, \lambda \in C-R .
$$

By $P_{j}$ we denote the orthogonal projection from $\mathfrak{S}_{1} \oplus \mathfrak{S}_{2}$ onto $\mathfrak{S}_{j}$ and by $P_{j}^{(2)}$ we denote the orthogonal projection from $\left(\mathfrak{S}_{1} \oplus \mathfrak{S}_{2}\right)^{2}$ onto $\mathfrak{S}_{j}^{2}$ defined by

$$
P_{\jmath}^{(2)}\{f, g\}=\left\{P_{j} f, P_{j} g\right\},\{f, g\} \in\left(\mathscr{S}_{1} \oplus \mathscr{S}_{2}\right)^{2}
$$

for $j=1,2$. Using these notations we state the following theorem.

THEOREM 6.3. Let $S_{1}$ be a symmetric subspace in $\mathfrak{\mathcal { C }}_{1}^{2}$. Let $S_{1} \subset$ $H$, where $H$ is a self-adjoint subspace in $\AA^{2}, \Re=\mathfrak{S}_{1} \oplus \mathfrak{S}_{2}$. Let $S_{2}=$ $H \cap \mathfrak{S}_{2}^{2}$ and let $\lambda \in \boldsymbol{C}-\boldsymbol{R}$ be fixed. Then $S_{2}$ is a symmetric subspace in $\mathfrak{S}_{2}^{2}$ such that

$$
\begin{aligned}
\operatorname{dim} M_{\lambda}^{2} & \leqq \operatorname{dim} M_{\bar{\lambda}}^{1}, \\
\operatorname{dim}\left(M_{\lambda}^{1} \oplus M_{\lambda}^{2}\right) & =\operatorname{dim}\left(M_{\bar{\lambda}}^{1} \oplus M_{\bar{\lambda}}^{2}\right),
\end{aligned}
$$

and $H$ can be written in the form

$$
H=S_{1} \oplus S_{2}+(I-V) M_{\lambda},
$$

where $V$ is an isometry in $\left(\mathfrak{S}_{1} \oplus \mathfrak{F}_{2}\right)^{2}$ such that

$$
\begin{gathered}
\mathfrak{D}(V)=M_{\lambda} \text { and } \Re(V)=M_{\bar{\lambda}}, \\
P_{1}^{(2)} V \text { maps } M_{\bar{\lambda}}^{2} \text { one to one into } M_{\bar{\lambda}}^{1},
\end{gathered}
$$

or equivalently

$$
P_{2}^{(2)} V^{-1} \operatorname{maps} M_{\bar{\lambda}}^{1} \text { onto } M_{\lambda}^{2} \text {. }
$$

Conversely, suppose that for a given symmetric subspace $S_{1}$ in $\mathfrak{S}_{1}^{2}$ there are a Hilbert space $\mathscr{F}_{2}$, a symmetric subspace $S_{2}$ in $\mathfrak{S}_{2}^{2}$ satisfying (6.6) and (6.7) for a fixed $\lambda \in \boldsymbol{C}-\boldsymbol{R}$ and an isometric operator $V$ in $\Re^{2}, \Re=\mathfrak{F}_{1} \oplus \mathscr{\mathcal { S }}_{2}$ satisfying (6.9) and (6.10). Then $H$ defined by (6.8) is a self-adjoint subspace in $\Omega^{2}$ and $S_{2}$ has the property that $S_{2}=H \cap \mathfrak{S}_{2}^{2}$. For $\lambda \in\{i,-i\}$ the direct sum in (6.8) is orthogonal. 
Using the Cayley transform we can deduce this theorem from Theorem 5.2 in a similar way as Theorem 6.2 is deduced from Theorem 5.1. We omit the details.

COROLLARY 6.4. Let $S$ be a symmetric subspace in $\mathfrak{S}^{2}$. Then $S$ has self-adjoint extensions in $\mathfrak{S}^{2}$ if and only if for some $\lambda \in \boldsymbol{C}-\boldsymbol{R}$

$$
\operatorname{dim} M_{\lambda}=\operatorname{dim} M_{\bar{\lambda}}
$$

If (6.12) holds, then all self-adjoint extensions $H$ of $S$ in $\mathfrak{S}^{2}$ are of the form

$$
H=S+(I-V) M_{\lambda},
$$

where $V$ is an isometry from $M_{\lambda}$ onto $M_{\bar{\lambda}}$. Conversely, each such isometry determines a self-adjoint extension of $S$ in $\mathfrak{S}^{2}$ by (6.13). For $\lambda \in\{i,-i\}$ the direct sum in (6.13) is orthogonal.

Let $S$ be a symmetric subspace in $\mathfrak{S C}^{2}$. Let $H$ be a self-adjoint subspace extension of $S$ in $\Re^{2}, \mathfrak{S} \subset \Re$. We call the extension $H$ minimal if $C_{i}(H)$ is a minimal unitary extension of $C_{i}(S)$. From the remarks following Corollary 5.3 and Theorem 4.3 it follows that $H$ is minimal if and only if $\Omega$ is spanned by

$$
\{E(t) f / f \in \mathfrak{S}, t \in R\} \cup \mathfrak{S}
$$

where $E$ is the spectral function of $H$ in $\Re^{2}$ (cf. Theorems 4.1 and 4.2). Furthermore, it follows that each self-adjoint extension of a symmetric subspace contains a minimal extension.

THEOREM 6.5. Let $S$ be a symmetric subspace in $\mathfrak{S}^{2}$. For $j=$ 1,2 let $H_{j}$ be a minimal self-adjoint subspace extension of $S$ in $\Re_{j}^{2}$ such that

$$
P_{1} E_{1}(t)=P_{2} E_{2}(t) \text { on } \mathfrak{S}, t \in \boldsymbol{R},
$$

where $E_{j}$ is the spectral function of $H_{j}$ in $\boldsymbol{\Omega}_{j}^{2}$ and $P_{j}$ is the orthogonal projection from $\Re_{j}$ onto $\mathfrak{S}$. Then there exists an isomorphism $\Phi$ from $\Re_{1}$ onto $\Re_{2}$ such that $\Phi f=f$ for all $f \in \mathscr{S}$ and $\Phi^{(2)} H_{1}=H_{2}$, where $\Phi^{(2)}: \Re_{1}^{2} \rightarrow \AA_{2}^{2}$ is defined by

$$
\Phi^{(2)}\{h, k\}=\{\Phi h, \Phi k\},\{h, k\} \in \mathcal{N}_{1}^{2} .
$$

Proof. Let $V=C_{i}(S)$ and $U_{j}=C_{i}\left(H_{j}\right), j=1,2$. Then on account of Theorem 4.3 the relation (6.14) implies (5.13) and hence (5.12) holds. Thus the conditions of Theorem 5.4 are satisfied, and the isomorphism $\Phi$ of Theorem 5.4 satisfies the conclusions of this theorem. 
We shall now describe the self-adjoint extensions $H$ in $\Re^{2}$ of a symmetric subspace $S$ in $\mathscr{S}_{2}^{2}$ in another way, analogous to the results of Straus [12] for operator extensions. To this end let $P$ be the orthogonal projection of $\Re$ onto $\mathfrak{S}_{\mathcal{C}}$ and denote by $P^{(2)}$ the mapping $P^{(2)}\{h, k\}=\{P h, P k\},\{h, k\} \in \Re^{2}$. It is not difficult to see that $S \subset P^{(2)} H \subset S^{*}$.

THEOREM 6.6. Let $S$ be a symmetric subspace in $\mathfrak{S c}^{2}$. Let $H$ be a self-adjoint subspace extension of $S$ in $\Re^{2}, \mathfrak{S} \subset \Re$. For $\lambda \in C-\boldsymbol{R}$ let

$$
L(\lambda)=\left\{\{R(\lambda) h, \lambda R(\lambda) h+h\} / h \in \mathfrak{S}_{\mathfrak{C}}\right\},
$$

where $R(\lambda)=(H-\lambda)^{-1}$. Then $L(\lambda)$ is a symmetric subspace in $\mathfrak{R}^{2}$. Let

$$
T(\lambda)=P^{(2)} L(\lambda), \lambda \in \boldsymbol{C}-\boldsymbol{R}
$$

then $\boldsymbol{R}(\lambda)=(T(\lambda)-\lambda)^{-1}$, where $\boldsymbol{R}(\lambda)=\left.P R(\lambda)\right|_{\mathfrak{s}}$,

$$
S \subset T(\lambda) \subset S^{*}
$$

and $T \in \boldsymbol{T}(\mathfrak{S})$. Conversely, if $T \in \boldsymbol{T}(\mathfrak{K})$ satisfies (6.17) for a given symmetric subspace $S$ in $\mathfrak{S}^{2}$, then there exists a Hilbert space $\mathbb{R} \supset \mathfrak{F}$ and a self-adjoint subspace extension $H$ in $\mathfrak{K}^{2}$ of $S$, such that $T$ satisfies (6.16), where $L$ is defined by (6.15).

Proof. Since $L(\lambda) \subset H$, it is symmetric. From (6.16) it follows that

$$
T(\lambda)=\{\{R(\lambda) h, \lambda R(\lambda) h+h\} / h \in \mathfrak{S}\}
$$

where $R=\left.P R\right|_{\mathfrak{S}} \in \boldsymbol{R}(\mathfrak{S})$. According to Theorem 4.5 we find $T \in \boldsymbol{T}(\mathfrak{S})$. The verification of (6.17) is straightforward. The second part of the theorem follows from Theorem 4.8.

THEOREM 6.7. Let $S$ be a symmetric subspace in $\mathfrak{S c}^{2}$. Then

$$
T \in T(\mathfrak{S}) \text { and } S \subset T(\lambda) \subset S^{*} \text { for all } \lambda \in C-\boldsymbol{R}
$$

if and only if

$$
\begin{aligned}
& T(\lambda)=S+(I-V(\lambda)) M_{\bar{\mu}}, \\
& T(\bar{\lambda})=S+(I-V(\bar{\lambda})) M_{\mu},
\end{aligned}
$$

for all $\lambda \in C^{+}$and fixed $\mu \in C^{+}$, where $V(\lambda)$ is an analytic contraction, mapping $M_{\bar{\mu}}$ into $M_{\mu}$, i.e., (1) $(V(\lambda)\{h, k\},\{x, y\})$ is analytic in $C^{+}$for all $\{h, k\} \in M_{\bar{\mu}}^{-},\{x, y\} \in \mathfrak{S}^{2}$, (2) if $V(\lambda)\{h, k\}=\{f, g\}$ then $\|f\|^{2}+$ $\|g\|^{2} \leqq\|h\|^{2}+\|k\|^{2}$, and where $V(\bar{\lambda})$ maps $M_{\mu}$ into $M_{\bar{\mu}}$ and is determined by 


$$
(V(\bar{\lambda})\{f, g\},\{h, k\})=(\{f, g\}, V(\lambda)\{h, k\})
$$

for all $\{f, g\} \in M_{\mu},\{h, k\} \in M_{\bar{\mu}}$. (Hence $V(\bar{\lambda})$ is a contraction, analytic in $\left.\bar{\lambda} \in C^{-}\right)$). If $\mu=i$ the direct sums in (6.19) are orthogonal.

Proof. Let (6.18) be satisfied and let $\mu \in C^{-}$be fixed. Since $\operatorname{Im} T(\lambda) \leqq 0(\max )$ and $\operatorname{Im} T(\bar{\lambda}) \geqq 0(\max )$ for $\lambda \in C^{+}, C_{\mu}(T(\lambda))$ and $C_{\bar{\mu}}(T(\bar{\lambda}))$ are contractions defined on all of $\mathfrak{S}_{\mathrm{c}}$. From (6.18) we deduce that

$$
\begin{aligned}
& C_{\mu}(T(\lambda))=C_{\mu^{\prime}}(S)+W(\lambda), \\
& C_{\bar{u}}^{-}(T(\bar{\lambda}))=C_{\bar{l}}^{-}(S)+W(\bar{\lambda}),
\end{aligned}
$$

where $W(\lambda)$ is the restriction of $C_{\mu}(T(\lambda))$ to $\mathfrak{S} \ominus \mathfrak{D}\left(C_{\mu}(S)\right)=\mathfrak{D}\left(M_{\bar{\mu}}\right)$ and $W(\bar{\lambda})$ is the restriction of $C_{\bar{\mu}}(T(\bar{\lambda}))$ to $\mathfrak{S} \ominus \mathscr{D}\left(C_{\bar{\mu}}(S)\right)=\mathfrak{D}\left(M_{\mu}\right)$. Hence $W(\lambda)$ is an analytic contraction for $\lambda \in C^{+}$and $W(\bar{\lambda})$ is an analytic contraction for $\bar{\lambda} \in \boldsymbol{C}^{-}$. From $\left(C_{\mu}(T(\lambda))\right)^{*}=C_{\bar{\mu}}(T(\bar{\lambda})$ ) (cf. Lemma 2.6), $\mathfrak{R}\left(C_{\bar{\mu}}(S)\right)=\mathfrak{S} \ominus \mathfrak{D}\left(M_{l}\right)$ and $\mathfrak{R}\left(C_{\mu^{\prime}}(S)\right)=\mathfrak{S} \ominus \mathfrak{D}\left(M_{\bar{\mu}}\right)$ we derive

$$
\begin{aligned}
& \Re(W(\lambda)) \subset \mathfrak{D}\left(M_{\mu}\right), \\
& \Re(W(\bar{\lambda})) \subset \mathfrak{D}\left(M_{\bar{\prime}}\right), \\
& (W(\lambda) x, y)=(x, W(\bar{\lambda}) y) \text { for all } x \in \mathfrak{D}\left(M_{\bar{\prime}}\right), y \in \mathfrak{D}\left(M_{\mu}\right) .
\end{aligned}
$$

Define

$$
V(\lambda)\{x, \bar{\mu} x\}=\{W(\lambda) x, \mu W(\lambda) x\}, x \in \mathcal{D}\left(M_{\bar{\mu}}\right),
$$

and

$$
V(\bar{\lambda})\{y, \mu y\}=\{W(\bar{\lambda}) y, \bar{\mu} W(\bar{\lambda}) y\}, y \in \mathfrak{D}\left(M_{\mu}\right) .
$$

Then $V(\lambda)$ and $V(\bar{\lambda})$ satisfy the descriptions in (6.19) and

$$
\begin{aligned}
& F_{\mu}(W(\lambda))=(I-V(\lambda)) M_{\mu}, \\
& F_{\bar{\mu}}(W(\bar{\lambda}))=(I-V(\bar{\lambda})) M_{\bar{\mu}} .
\end{aligned}
$$

Applying $F_{\mu \prime}$ to (6.20) and $F_{\bar{\mu}}$ to $(6.21)$ and using the last two equalities we find the relations (6.19).

Now lot $(6.19)$ be satisfied. Define $W(\lambda)$ and $W(\bar{\lambda})$ by $(6.23)$ and (6.24). Then $W(\lambda)$ is an analytic contraction, mapping $\mathscr{D}\left(M_{\mu}\right)$ into $D\left(M_{\mu}\right)$ and since (6.20) also holds we conclude that $C_{\mu}(T(\lambda))$ is an analytic contraction defined on all of $\mathfrak{S}$. Hence $\operatorname{Im} T(\lambda) \leqq 0(\max )$. Also (6.21) and (6.22) hold, and since $S$ is symmetric we have

$$
C_{\bar{\mu}}(T(\bar{\lambda})) \subset\left(C_{\mu}(T(\lambda))\right)^{*}=C_{\bar{\mu}}\left((T(\lambda))^{*}\right) .
$$

Now $\mathfrak{D}\left(C_{\bar{\mu}}^{-}(T(\bar{\lambda}))\right)=\mathfrak{S}$ and since $C_{\bar{\mu}}^{-}(T(\bar{\lambda}))$ and $C_{\bar{\mu}}^{-}\left((T(\lambda))^{*}\right)$ are operators 
they are equal. Applying $F_{\bar{\mu}}$ we find $T(\lambda)^{*}=T(\bar{\lambda})$. Hence $T \in \boldsymbol{T}(\mathfrak{S})$. Clearly $S \subset T(\lambda) \subset S^{*}$. Hence (6.18) holds.

Another way of describing all self-adjoint extensions $H$ in $\Re^{2}$ of a symmetric subspace $S$ in $\mathfrak{S C}^{2}$ can be given via the class $R(\mathfrak{S})$, cf. McKelvey [6].

Theorem 6.8. Let $S$ be a symmetric subspace in $\mathfrak{S c}^{2}$. Let $H$ be a self-adjoint subspace extension of $S$ in $\Re^{2}, \mathfrak{S} \subset \Re$. For $\lambda \in \boldsymbol{C}-\boldsymbol{R}$ let

$$
\mathbf{R}(\lambda)=\left.P R(\lambda)\right|_{\tilde{5}}
$$

where $R(\lambda)=(H-\lambda)^{-1}$ and $P$ is the orthogonal projection of $\Re$ onto Sc. Then $\boldsymbol{R} \in \boldsymbol{R}(\mathfrak{S})$ and

$$
\boldsymbol{R}(\lambda)(S-\lambda) \subset I, \lambda \in \boldsymbol{C}-\boldsymbol{R} .
$$

Conversely, suppose that for some $\boldsymbol{R} \in \boldsymbol{R}(\mathfrak{S})$ (6.26) holds. Then there exists a Hilbert space $\mathfrak{R} \supset \mathfrak{S}$ and a self-adjoint subspace extension $H$ of $S$ in $\Re^{2}$, such that $\mathbf{R}$ satisfies (6.25) for $\lambda \in \boldsymbol{C}-\boldsymbol{R}$.

Proof. Let $\boldsymbol{R}$ be defined by $(6.25)$, then clearly $\boldsymbol{R} \in \boldsymbol{R}(\mathfrak{S})$. Let $T(\lambda)=\lambda+\boldsymbol{R}(\lambda)^{-1}$. Then $T \in \boldsymbol{T}(\mathfrak{\mathcal { C }})$ by Theorem 4.5 and $T$ satisfies (6.16). Hence by Theorem 6.6 the assertion (6.17) holds. But (6.17) is equivalent to (6.26). The converse follows in a similar way from the second part of Theorem 6.6.

Related to Theorem 6.8 is the following result, the operator version of which is due to McKelvey [6]. The proof follows the same lines as in [6].

THEOREM 6.9. Let $S$ be a symmetric subspace in $\mathfrak{S}^{2}$. Let $\boldsymbol{R} \in \boldsymbol{R}(\mathfrak{S})$ and $T \in \boldsymbol{T}(\mathfrak{K})$ be connected by $T(\lambda)=\lambda+\boldsymbol{R}(\lambda)^{-1}, \lambda \in \boldsymbol{C}-\boldsymbol{R}$. Suppose for some $\lambda \in \boldsymbol{C}-\boldsymbol{R}$

$$
\boldsymbol{R}(\lambda)(S-\lambda) \subset I
$$

or equivalently

$$
S \subset T(\lambda)
$$

Then

(i) if $\lambda \in C^{+}\left(\lambda \in C^{-}\right)$then $\operatorname{Im} S \leqq 0(\operatorname{Im} S \geqq 0)$,

(ii) if (6.27) holds for some $\lambda \in C^{+}$and for some $\lambda \in C^{-}$, then $S$ is symmetric and (6.27) (or equivalently (6.28)) holds for all $\lambda \in \boldsymbol{C}-\boldsymbol{R}$.

Finally we return to the situation as described in Theorem 6.6. Hence let $S$ be a symmetric subspace in $\mathfrak{S}^{2}$ and let $H$ be a self- 
adjoint subspace extension of $S$ in $\Re^{2}$. The subspace $H$ defines a function $T \in T(\mathscr{S})$ such that $S \subset T(\lambda) \subset S^{*}(\lambda \in C-R)$. The function $T \in \boldsymbol{T}(\mathfrak{S})$ gives rise to a function $T_{s} \in \boldsymbol{T}_{s}(\mathfrak{S} \ominus T(0))$ according to Theorem 4.7. On the other hand, the self-adjoint subspace $H$ in $\AA^{2}$ defines a densely defined self-adjoint operator $H_{s}$ in $\Re \ominus H(0)$.

LeMma 6.10. (i) $T(0)=\mathfrak{S} \cap H(0)$,

(ii) $T(0) \subset P H(0)$,

(iii) $(\mathfrak{S} \ominus T(0)) \cap(\Re \ominus H(0))=\mathscr{S} \ominus P H(0)$,

(iv) $P(\Re \ominus H(0)) \subset \mathfrak{S} \ominus T(0)$.

Proof. Let $h \in T(0)$, then $\{0, h\} \in T(\lambda)$, hence $h \in \mathfrak{S}$ and $P R(\lambda) h=$ 0 , where $R(\lambda)=(H-\lambda)^{-1}, \lambda \in \boldsymbol{C}-\boldsymbol{R}$. This implies $h \in \mathfrak{S}$ and $R(\lambda) h \perp \mathfrak{F}$. Now

$$
\{R(\lambda) h, \lambda R(\lambda) h+h\} \in H
$$

shows

$$
\bar{\lambda}\|R(\lambda) h\|^{2}+(R(\lambda) h, h)=(h, R(\lambda) h)+\lambda\|R(\lambda) h\|^{2} .
$$

Hence $R(\lambda) h=0$, while $h \in \mathscr{F}$, thus $h \in \mathscr{S} \cap H(0)$. Therefore $T(0) \subset$ $\mathfrak{S} \cap H(0)$, while the other inclusion is clear. This proves (i). A consequence of (i) is $T(0) \subset H(0)$, which implies (ii). Hence $\mathfrak{S} \ominus P H(0) \subset$ $\mathfrak{S} \ominus T(0)$. Now

$$
(\mathfrak{S} \ominus T(0)) \cap(\Re \ominus H(0))=(\mathfrak{S} \ominus T(0)) \cap \mathfrak{F} \cap\left(H(0)^{\perp}\right)
$$

and $\mathfrak{S} \cap\left(H(0)^{\perp}\right)=\mathfrak{S} \ominus P H(0)$. This proves (iii). Let $h \in \Re \ominus H(0)$, then $P h \in \mathfrak{S}$ and $(h, u)=0$ for all $h \in H(0)$, hence certainly $(h, u)=0$ for all $u \in \mathfrak{S} \cap H(0)=T(0)$. This proves (iv).

We have already noted that $S \subset P^{(2)} H \subset S^{*}$, but then also $P^{(2)} H_{\infty} \subset$ $S^{*}$ or $P H(0) \subset S^{*}(0)$. In case $S$ is a densely defined symmetric operator in $\mathfrak{S}$, then this inclusion shows $H(0) \perp \mathfrak{S}$, and by Lemma 6.10 we find $T(0)=\{0\}$. Hence $T$ is a function, with densely defined operators as values. It is clear that a densely defined symmetric operator $S$ has self-adjoint operator extensions: If $H$ is a self-adjoint subspace extension in $\Omega$, then $H_{s}$ is a self-adjoint operator extension in $\mathscr{R} \ominus H(0)$. Any function $\boldsymbol{E} \in \boldsymbol{E}(\mathfrak{F})$ which is associated with $T \in \boldsymbol{T}(\mathfrak{F})$ via Theorems 4.5 and 4.6 has the property that $E(\infty)$ is the identity on $\mathfrak{S}$.

The situation may be quite different if $S$ is any symmetric subspace in $\mathfrak{S}^{2}$. The self-adjoint extension $H$ in $\Re^{2}$ generates a densely defined self-adjoint operator $H_{s}$ in $\Re \ominus H(0)$; also $H$ gives rise to a function $T \in T(\mathfrak{S})$ according to Theorem 6.6. This function $T \in T(\mathfrak{E})$ itself generates an operator-valued function $T_{s} \in T_{s}(\mathfrak{S} \ominus T(0))$, accord- 
ing to Theorem 4.7. Let $E_{s}$ be the restriction to $\Re \ominus H(0)$ of the spectral function $E \in \mathbf{E}(\Re)$ belonging to $H$ via Theorems 4.1 and 4.2 , and let $\boldsymbol{E}_{\boldsymbol{s}}$ be the restriction to $\mathfrak{S} \ominus T(0)$ of the spectral function $\boldsymbol{E} \in \boldsymbol{E}(\mathfrak{S})$ belonging to the function $T \in \boldsymbol{T}(\mathfrak{S})$ via Theorems 4.5 and 4.6. The relation

$$
\boldsymbol{E}(t)=\left.P E(t)\right|_{\mathfrak{s}}, t \in \boldsymbol{R},
$$

is easily established, since $R(\lambda)=\left.P R(\lambda)\right|_{\mathfrak{s}}, \lambda \in \boldsymbol{C}-\boldsymbol{R}$ (cf. the proof of Theorem 6.6). From (6.29) we conclude

$$
\boldsymbol{E}_{s}(t)=\left.P E(t)\right|_{\mathfrak{n} \ominus T(0)},
$$

while we already have

$$
P E_{s}(t)=\left.P E(t)\right|_{\Re \ominus H(0)} \text {. }
$$

The assertions (6.30) and (6.31), together with Lemma 6.10 show that on $\mathfrak{S} \ominus P H(0)$ the functions $E_{s}$ and $P E_{s}$ coincide. $^{1}$

\section{REFERENCES}

1. N. I. Achieser and I. M. Glasmann, Theorie der linearen Operatoren im HilbertRaum, Akademie-Verlag, Berlin, 1968.

2. R. Arens, Operational calculus of linear relations, Pacific J. Math., 11 (1961), 9-23.

3. C. Bennewitz, Symmetric Relations on a Hilbert Space, in Conference on the theory of ordinary and partial differential equations, Lecture Notes in Mathematics, 280 Springer, Berlin, 1972.

4. E. A. Coddington, Extension theory of formally normal and symmetric subspaces, Mem. Amer. Math. Soc., 134 (1973).

5. - Self-adjoint problems for non-densely defined ordinary differential operators and their eigenfunction expansions, Advances in Math., 14 (1974).

6. R. McKelvey, Spectral measures, generalized resolvents and functions of positive type, J. Math. Anal. Appl., 11 (1965), 447-477.

7. M. A. Naimark, Spectral functions of a symmetric operator, Izv. Akad. Nauk SSSR. Ser. Mat., 4 (1940), 277-318.

8. - On spectral functions of a symmetric operator, Izv. Akad. Nauk SSSR. Ser. Mat., 7 (1943), 285-296.

9. — Linear Differential Operators, Part 2, Harrap, London, 1968.

10. R. S. Phillips, Dissipative operators and hyperbolic systems of partial differential equations, Trans. Amer. Math. Soc., 90 (1959), 193-254.

11. A. Schneider, Zum Entwicklungssatz bei reellen singulären Differential-glechungssystemen, Arch. Math., 21 (1970), 192-197.

12. A. V. Štraus, Generalized resolvents of symmetric operators, Izv. Akad. Nauk SSSR. Ser. Mat., 18 (1954), 51-86.

13. B. Sz.-Nagy and C. Foias, Harmonic Analysis of Operators on Hilbert Space,

1 Coddington [5] introduces the function $F_{s}$ by

$$
F_{s}=\left.P E_{s}\right|_{s \ominus P H(0)}
$$

and calls this function a generalized spectral family for $S$ corresponding to $H$ (and $H_{s}$ ). Hence $F_{s}$ is the restriction of $\boldsymbol{E}_{\boldsymbol{s}}$ to $\mathfrak{g} \Theta P H(0)$, with the additional property

$$
F_{s}(\infty) f=f, f \in \mathscr{H} \ominus P H(0) \text {. }
$$


North-Holland Publishing Company, Amsterdam-London, 1970.

Received November 1, 1973.

University of CAlifornia, Los Angeles

AND

Rijksuniversiteit Groningen, Nederland 


\section{PACIFIC JOURNAL OF MATHEMATICS}

\section{EDITORS}

RICHARD ARENS (Managing Editor)

University of California

Los Angeles, California 90024

R. A. Beaumont

University of Washington

Seattle, Washington 98105
J. DugundJI

Department of Mathematics University of Southern California Los Angeles, California 90007

D. Gilbarg AND J. Milgram Stanford University

Stanford, California 94305

\section{ASSOCIATE EDITORS}
E. F. BECKENBACH
B. H. NeUmanN
F. WOLF
K. YOSHIDA

\section{SUPPORTING INSTITUTIONS}

UNIVERSITY OF BRITISH COLUMBIA CALIFORNIA INSTITUTE OF TECHNOLOGY

UNIVERSITY OF CALIFORNIA

MONTANA STATE UNIVERSITY

UNIVERSITY OF NEVADA

NEW MEXICO STATE UNIVERSITY

OREGON STATE UNIVERSITY

UNIVERSITY OF OREGON

OSAKA UNIVERSITY
UNIVERSITY OF SOUTHERN CALIFOF

STANFORD UNIVERSITY

UNIVERSITY OF TOKYO

UNIVERSITY OF UTAH

WASHINGTON STATE UNIVERSITY UNIVERSITY OF WASHINGTON

AMERICAN MATHEMATICAL SOCIET NAVAL WEAPONS CENTER 


\section{Pacific Journal of Mathematics}

\section{Vol. 54, No. 1 \\ May, 1974}

Ralph K Amayo, Engel Lie rings with chain conditions ..................

Bernd Anger and Jörn Lembcke, Hahn-Banach type theorems for hypolinear

functionals on preordered topological vector spaces ..................

Gregory Frank Bachelis and Samuel Ebenstein, On $\Lambda(p)$ sets ................

Harvey Isaac Blau, Indecomposable modules for direct products of finite

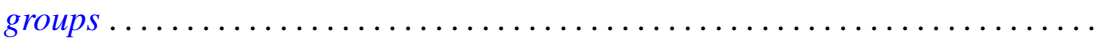

Larry Eugene Bobisud and James Calvert, Singular perturbation of a

time-dependent Cauchy problem in a Hilbert space ................

Walter D. Burgess and Robert Raphael, Abian's order relation and orthogonal

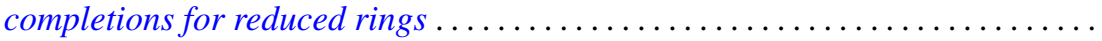

James Diederich, Representation of superharmonic functions mean continuous at

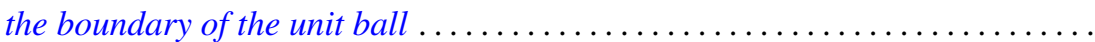

Aad Dijksma and Hendrik S. V. de Snoo, Self-adjoint extensions of symmetric

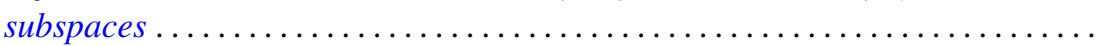

Gustave Adam Efroymson, A Nullstellensatz for Nash rings . . . . . . . . . . . . .

John D. Elwin and Donald R. Short, Branched immersions onto compact orientable surfaces . . . . . . . . . . . . . . . . . . . . . . . . .

John Douglas Faires, Comparison of the states of closed linear

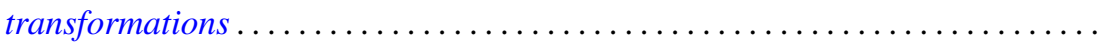

Joe Wayne Fisher and Robert L. Snider, On the von Neumann regularity of rings with regular prime factor rings .

Franklin Takashi Iha, A unified approach to boundary value problems on compact intervals

Palaniappan L. Kannappan and Che Tat $\mathrm{Ng}$, On functional equations connected with directed divergence, inaccuracy and generalized directed divergence

Samir A. Khabbaz and Elias Hanna Toubassi, The module structure of Ext $(F, T)$ over the endomorphism ring of $T$...

Garo K. Kiremidjian, On deformations of complex compact manifolds with boundary.

Dimitri Koutroufiotis, Mappings by parallel normals preserving principal

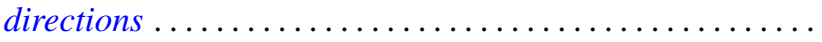

W. K. Nicholson, Semiperfect rings with abelian adjoint group

Norman R. Reilly, Extension of congruences and homomorphisms to translational hulls

Sadahiro Saeki, Symmetric maximal ideals in $M(G)$

Brian Kirkwood Schmidt, On the homotopy invariance of certain functors ...

H. J. Shyr and T. M. Viswanathan, On the radicals of lattice-ordered rings ...

Indranand Sinha, Certain representations of infinite group algebras ...

David Smallen, The group of self-equivalences of certain complexes ...

Kalathoor Varadarajan, On a certain problem of realization in homotopy

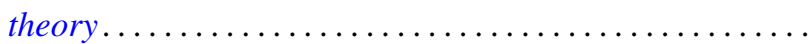

